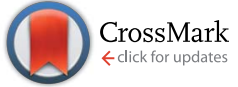

Cite this: RSC Adv., 2014, 4, 34801

\title{
Factors controlling the photoresponse of copper(I) diimine dyes containing hole-transporting dendrons in dye-sensitized solar cells: substituent and solvent effects $\dagger$
}

\author{
Sven Y. Brauchli, Biljana Bozic-Weber, Edwin C. Constable, ${ }^{\star}$ Nik Hostettler, \\ Catherine E. Housecroft* and Jennifer A. Zampese
}

\begin{abstract}
Two series of 2,2'-bipyridine (bpy) ligands bearing different 6,6'-substituents ( $\mathrm{Me},{ }^{n} \mathrm{Bu}$, iso $\mathrm{Bu}$, hexyl, $\mathrm{Ph}$ and 2-naphthyl) and carrying first-generation (ligands 1-6) or second-generation (ligands 7-12) hole transporting dendrons in the $4,4^{\prime}$-positions are reported. They have been incorporated into homoleptic copper(I) complexes $\left[\mathrm{CuL}_{2}\right]\left[\mathrm{PF}_{6}\right]$. $\mathrm{FTO} / \mathrm{TiO}_{2}$ electrodes functionalized with the anchoring ligand $\left(\left(6,6^{\prime}-\right.\right.$ dimethyl-[2,2' -bipyridine]-4,4'-diyl)bis(4,1-phenylene))bis(phosphonic acid), 13, were dipped in either $\mathrm{CH}_{2} \mathrm{Cl}_{2}$ or acetone solutions of $\left[\mathrm{CuL}_{2}\right]\left[\mathrm{PF}_{6}\right]$ to produce two series of surface-bound heteroleptic dyes. Their performances in dye-sensitized solar cells (DSCs) are assessed. Solid-state absorption spectra of dye-functionalized electrodes show that dye uptake is greater if acetone is used in the dye-dipping cycle rather than $\mathrm{CH}_{2} \mathrm{Cl}_{2}$, and the DSCs made using acetone generally perform better than analogous DSCs made using $\mathrm{CH}_{2} \mathrm{Cl}_{2}$. Using acetone-dipping solutions, the best DSC efficiencies are obtained with the second-generation dyes $[\mathrm{Cu}(13)(\mathrm{L})]^{+}\left(\mathrm{L}=7-11\right.$ with $\mathrm{Me},{ }^{n} \mathrm{Bu},{ }^{\text {iso }} \mathrm{Bu}$, hexyl, Ph groups); $[\mathrm{Cu}(13)(12)]^{+}(12$ contains 2-naphthyl groups in the 6,6'-positions) and its first-generation analogue $[\mathrm{Cu}(13)(6)]^{+}$perform poorly. When $\mathrm{CH}_{2} \mathrm{Cl}_{2}$ is used in the dipping cycle, DSCs with dyes $[\mathrm{Cu}(13)(1)]^{+}$and $[\mathrm{Cu}(13)(7)]^{+}\left(6,6^{\prime}-\mathrm{Me}_{2^{-}}\right.$ substituted) show the highest $V_{\mathrm{OC}}, J_{\mathrm{SC}}$ and $\eta$ values, and EQE spectra confirm electron injection over a wider energy range than for other dyes. For $\mathrm{CH}_{2} \mathrm{Cl}_{2}$ in the dipping cycle (but not for acetone), $[\mathrm{Cu}(13)(5)]^{+}\left(6,6^{\prime}-\mathrm{Ph}_{2}\right.$-substituted) performs as well as $[\mathrm{Cu}(13)(1)]^{+}$. The overall results of the study indicate that a combination of small $6,6^{\prime}$-substituents and acetone in the dye-dipping cycle lead to the best performing dyes.
\end{abstract}

Received 23rd April 2014 Accepted 23rd July 2014

DOI: $10.1039 / c 4 r a 03700 f$

www.rsc.org/advances

\section{Introduction}

Conventional Grätzel dye-sensitized solar cells (DSCs) incorporate ruthenium(II) complexes as photosensitizers. ${ }^{1}$ Our ongoing focus on DSCs containing copper(I) complexes as sensitizers is predicated both upon their possessing similar photophysical properties to ruthenium(II) complexes, ${ }^{2,3}$ and upon the greater abundance of copper than ruthenium in the Earth. Sauvage and co-workers pioneered the introduction of copper(I) complexes in DSCs ${ }^{4}$ and more recent uses of copper(I) in dyes in DSCs have been surveyed by Robertson ${ }^{5}$ and by us. ${ }^{6}$ The recent report of a remarkable photoconversion efficiency of $4.66 \%$ for a DSC with

Department of Chemistry, University of Basel, Spitalstrasse 51, CH4056 Basel, Switzerland. E-mail: edwin.constable@unibas.ch; catherine.housecroft@unibas.ch; Tel: +41612671008

$\dagger$ Electronic supplementary information (ESI) available: Syntheses and characterization of 4,4'-bis(4-bromophenyl)-6,6'-di(naphthalen-2-yl)-2,2'-bipyridine and ligands 2-6, 8-12; Fig. S1. SEM image of commercial electrode. Fig. S2-S6: additional $J-V$ curves and EQE spectra; Tables S1-S4: DSC parameters for all cells including duplicate data sets. See DOI: c4ra03700f a heteroleptic copper(I) dye containing 6,6'-dimesityl-2,2'bipyridine-4, $4^{\prime}$-dicarboxylic acid as the anchoring ligand and a $2,2^{\prime}$-bipyridine ancillary ligand with triphenylamino domains underlines the potential of copper(I) sensitizers. ${ }^{7}$ In the latter case, the efficiency was enhanced by using the co-adsorbant chenodeoxycholic acid.

We recently described the syntheses and characterization of the two copper(I) diimine complexes incorporating ligands containing first- and second-generation hole-transport dendrons (Scheme 1). For both ligands, semi-empirical MO calculations at the PM3 level showed that the HOMOs (degenerate set) and LUMO are localized on the dendron and 2,2'-bipyridine (bpy) metal-binding unit, respectively. ${ }^{8}$ By applying a ligand exchange strategy, ${ }^{9}$ we assembled dye-sensitized solar cells (DSCs) containing dyes $\left[\mathrm{Cu}\left(\mathrm{L}_{\text {ancillary }}\right)\left(\mathrm{L}_{\text {anchor }}\right)\right]^{+}$anchored on mesoporous $\mathrm{TiO}_{2}$. The combination of the hole-transport substituents in the ancillary ligands with the anchoring ligand 6,6'-dimethyl-2,2'-bipyridine-4,4'-bis(phosphonic acid) resulted in power-to-current conversion efficiencies of $20.7 \%$ relative to $100 \%$ for N719 measured under the same conditions in fully 


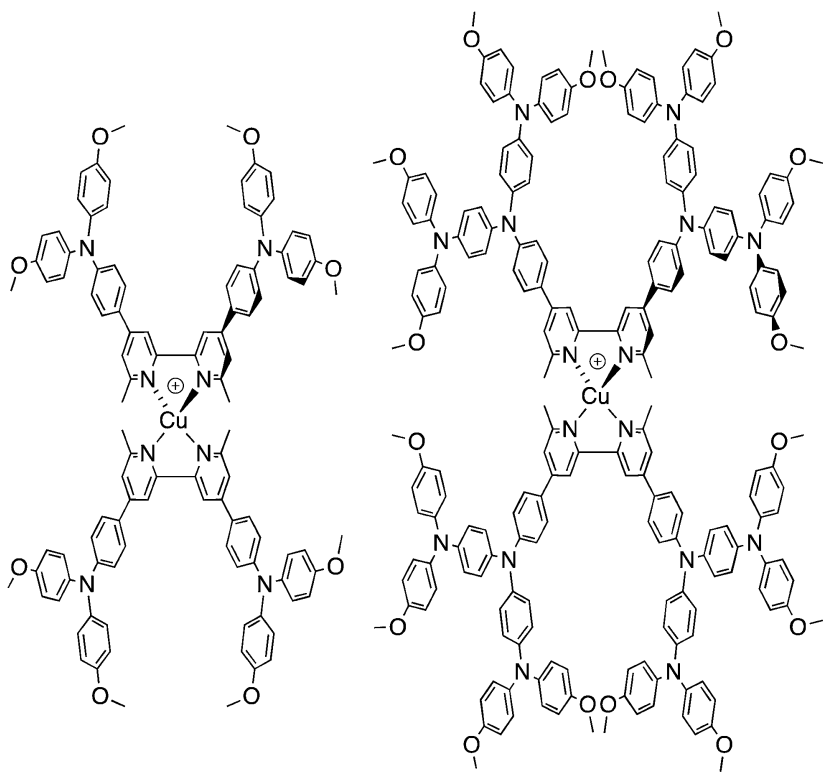

Scheme 1 Homoleptic copper(I) diimine complexes with ligands containing first- and second-generation hole-transport dendrons. ${ }^{8}$

masked DSCs. ${ }^{8}$ We have also established that the photoresponse of a $\left[\mathrm{Cu}\left(\mathrm{L}_{\text {ancillary }}\right)\left(\mathrm{L}_{\text {anchor }}\right)\right]^{+}$dye can be significantly improved by introducing an aromatic linker between the bpy and phosphonate domains of the anchoring ligand, and by replacing the 6- and 6'-methyl substituents in the ancillary ligand by isobutyl or phenyl groups..$^{10}$ We now describe a systematic extension of these studies in which the ancillary bpy ligands (1-12 in Scheme 2) contain (i) first- or secondgeneration hole-transport dendrons and (ii) alkyl or aromatic substituents of varying steric bulk in the 6,6'-positions. The anchoring ligand in the $\left[\mathrm{Cu}\left(\mathrm{L}_{\text {ancillary }}\right)\left(\mathrm{L}_{\text {anchor }}\right)\right]^{+}$dyes is $\mathbf{1 3}$ (Scheme 3) which has shown the greatest potential in recent studies. The heteroleptic complex is assembled using ligand exchange between surface-anchored ligand $\mathrm{L}_{\text {anchor }}$ and a homoleptic complex $\left[\mathrm{Cu}\left(\mathrm{L}_{\text {ancillary }}\right)_{2}\right]^{+}$. This approach circumvents the need to isolate the heteroleptic species (which is often not possible because of the rapid establishment of statistical solution equilibria between homo- and heteroleptic species). Characterisation of related surface species has previously been carried out. ${ }^{9}$

\section{Experimental}

\section{General}

A Bruker Avance III-500 NMR spectrometer was used to record ${ }^{1} \mathrm{H}$ and ${ }^{13} \mathrm{C}$ NMR spectra, and chemical shifts were referenced to residual solvent peaks with respect to $\partial(\mathrm{TMS})=0 \mathrm{ppm}$. Solution absorption spectra were recorded with a Cary 5000 spectrophotometer and FT-IR spectra of solid samples on a Perkin Elmer UATR Two spectrometer. MALDI-TOF and electrospray ionization (ESI) mass spectra were recorded on Bruker Daltonics microflex and Bruker esquire $3000^{\text {plus }}$ instruments, respectively. Electrochemical measurements were made using a $\mathrm{CH}$ Instruments 900B potentiostat with glassy carbon, platinum wire and silver wire as the working, counter, and reference electrodes, respectively. Substrates were dissolved in HPLC grade $\mathrm{CH}_{2} \mathrm{Cl}_{2}\left(\mathrm{ca} .10^{-4}\right.$ to $10^{-5} \mathrm{~mol} \mathrm{dm}{ }^{-3}$ ) containing $0.1 \mathrm{~mol}$ $\mathrm{dm}^{-3}\left[{ }^{n} \mathrm{Bu}_{4} \mathrm{~N}\right]\left[\mathrm{PF}_{6}\right]$ as the supporting electrolyte; all solutions were degassed with argon. $\mathrm{Cp}_{2} \mathrm{Fe}$ was used as internal reference.

The external quantum efficiency (EQE) measurements were made using a Spe-Quest quantum efficiency instrument from Rera Systems (Netherlands) equipped with a $100 \mathrm{~W}$ halogen lamp (QTH) and a lambda 300 grating monochromator (Lot Oriel). The monochromatic light was modulated to $3 \mathrm{~Hz}$ using a chopper wheel (ThorLabs). The cell response was amplified with a large dynamic range IV converter (CVI Melles Griot) and then measured with a SR830 DSP Lock-In amplifier (Stanford Research).

Compounds 1 and $7,{ }^{8}$ anchoring ligand $13,{ }^{10}\left[\mathrm{Cu}(\mathrm{MeCN})_{4}\right]\left[\mathrm{PF}_{6}\right],{ }^{11}$ $\left[\mathrm{Cu}(\mathbf{1})_{2}\right]\left[\mathrm{PF}_{6}\right]^{8}$ and $\left[\mathrm{Cu}(7)_{2}\right]\left[\mathrm{PF}_{6}\right]^{8}$ were prepared as previously reported. The syntheses and characterization data of the ligands and the numbering scheme for the NMR assignments are given in the ESI.†

\section{$\left[\mathrm{Cu}(2)_{2}\right]\left[\mathrm{PF}_{6}\right]$}

$\left[\mathrm{Cu}(\mathrm{MeCN})_{4}\right]\left[\mathrm{PF}_{6}\right](35.2 \mathrm{mg}, 0.094 \mathrm{mmol})$ was reacted with 2 (165 mg, $0.189 \mathrm{mmol}$ ) in a mixture of $\mathrm{MeCN}(15 \mathrm{~mL})$ and $\mathrm{CH}_{2} \mathrm{Cl}_{2}$ $(15 \mathrm{~mL})$. The solution turned red immediately and was stirred overnight at room temperature. Then, the volume of the solvent was reduced in vacuo and the product was precipitated by addition of $\mathrm{Et}_{2} \mathrm{O}$. The solid was separated by filtration, was washed with $\mathrm{Et}_{2} \mathrm{O}$ and was dried in a stream of air. $\left[\mathrm{Cu}(2)_{2}\right]\left[\mathrm{PF}_{6}\right]$ was isolated as a red solid (145 mg, $0.074 \mathrm{mmol}, 78 \%) .{ }^{1} \mathrm{H}$ NMR $\left(500 \mathrm{MHz}, \mathrm{CDCl}_{3}\right) \partial / \mathrm{ppm}: 8.27\left(\mathrm{~d}, J=1.2 \mathrm{~Hz}, 4 \mathrm{H}, \mathrm{H}^{\mathrm{A3}}\right), 7.59(\mathrm{~d}$, $\left.J=8.8 \mathrm{~Hz}, 8 \mathrm{H}, \mathrm{H}^{\mathrm{B} 2}\right), 7.57\left(\mathrm{~d}, J=1.2 \mathrm{~Hz}, 4 \mathrm{H}, \mathrm{H}^{\mathrm{A5}}\right), 7.14(\mathrm{~d}, J=9.0$ $\left.\mathrm{Hz}, 16 \mathrm{H}, \mathrm{H}^{\mathrm{C} 2}\right), 7.02\left(\mathrm{~d}, J=8.8 \mathrm{~Hz}, 8 \mathrm{H}, \mathrm{H}^{\mathrm{B}}\right), 6.89(\mathrm{~d}, J=9.0 \mathrm{~Hz}$, $\left.16 \mathrm{H}, \mathrm{H}^{\mathrm{C} 3}\right), 3.83(\mathrm{~s}, 24 \mathrm{H}, \mathrm{OMe}), 2.62\left(\mathrm{t}, J=8.2 \mathrm{~Hz}, 8 \mathrm{H}, \mathrm{H}^{\mathrm{a}}\right), 1.44-$ $1.32\left(\mathrm{~m}, 8 \mathrm{H}, \mathrm{H}^{\mathrm{b}}\right), 0.90\left(\mathrm{tq}, J=7.4 \mathrm{~Hz}, 8 \mathrm{H}, \mathrm{H}^{\mathrm{c}}\right), 0.48(\mathrm{t}, J=7.4 \mathrm{~Hz}$, $\left.12 \mathrm{H}, \mathrm{H}^{\mathrm{d}}\right) .{ }^{13} \mathrm{C}$ NMR $\left(126 \mathrm{MHz}, \mathrm{CDCl}_{3}\right) \partial / \mathrm{ppm}: 161.5\left(\mathrm{C}^{\mathrm{A} 6}\right), 156.8$ $\left(\mathrm{C}^{\mathrm{C} 4}\right), 152.6\left(\mathrm{C}^{\mathrm{A} 2}\right), 150.8\left(\mathrm{C}^{\mathrm{B} 4}\right), 150.2\left(\mathrm{C}^{\mathrm{B} 1}\right), 139.8\left(\mathrm{C}^{\mathrm{C} 1}\right), 127.8$ $\left(\mathrm{C}^{\mathrm{B} 2}\right), 127.5\left(\mathrm{C}^{\mathrm{C} 2}\right), 127.3\left(\mathrm{C}^{\mathrm{A} 4}\right), 121.8\left(\mathrm{C}^{\mathrm{A} 5}\right), 119.4\left(\mathrm{C}^{\mathrm{B} 3}\right), 116.3$ $\left(\mathrm{C}^{\mathrm{A} 3}\right), 115.1\left(\mathrm{C}^{\mathrm{C} 3}\right), 55.7\left(\mathrm{O}^{\mathrm{Me}}\right), 39.8\left(\mathrm{C}^{\mathrm{a}}\right), 31.9\left(\mathrm{C}^{\mathrm{b}}\right), 22.7\left(\mathrm{C}^{\mathrm{C}}\right), 13.6$ $\left(\mathrm{C}^{\mathrm{d}}\right) \cdot \operatorname{IR}\left(\tilde{\nu} / \mathrm{cm}^{-1}\right): 3187(\mathrm{w}), 3036(\mathrm{w}), 2997(\mathrm{w}), 2952(\mathrm{w}), 2924(\mathrm{w})$, $2866(\mathrm{w}), 2853(\mathrm{w}), 2832(\mathrm{w}), 1595(\mathrm{~s}), 1501(\mathrm{~s}), 1462(\mathrm{~m}), 1440$ $(\mathrm{m}), 1321(\mathrm{~m}), 1237(\mathrm{~s}), 1195(\mathrm{~m}), 1179(\mathrm{~m}), 1102(\mathrm{~m}), 1031(\mathrm{~m})$, $838(\mathrm{~s}), 824(\mathrm{~s}), 728(\mathrm{~m}), 574(\mathrm{~m}), 557(\mathrm{~m}), 527(\mathrm{~m})$. ESI MS (m/z): $1813.8\left[\mathrm{M}-\mathrm{PF}_{6}\right]^{+}$, (calc. 1813.8). UV-VIS $\left(\mathrm{CH}_{2} \mathrm{Cl}_{2}, 1.0 \times 10^{-5}\right.$ mol dm $\left.{ }^{-3}\right): \lambda_{\max } / \mathrm{nm} 306\left(\varepsilon / \mathrm{dm}^{3} \mathrm{~mol}^{-1} \mathrm{~cm}^{-1} 67500\right), 386$ (60 400), 480 sh (27 500). Found: C, 71.69; H, 6.32; N, 5.52; $\mathrm{C}_{116} \mathrm{H}_{116} \mathrm{~N}_{8} \mathrm{O}_{8} \mathrm{CuPF}_{6}$ requires C, 71.13; H, 5.97; N, $5.72(\%)$.

\section{$\left[\mathrm{Cu}(3)_{2}\right]\left[\mathrm{PF}_{6}\right]$}

The method was as for $\left[\mathrm{Cu}(2)_{2}\right]\left[\mathrm{PF}_{6}\right]$ starting with $\left[\mathrm{Cu}(\mathrm{MeCN})_{4}\right]\left[\mathrm{PF}_{6}\right]$ (47.9 mg, $0.128 \mathrm{mmol})$ and $3(225 \mathrm{mg}, 0.257 \mathrm{mmol})$ was isolated as red solid $(105 \mathrm{mg}, 0.054 \mathrm{mmol}, 42 \%) .{ }^{1} \mathrm{H}$ NMR $(500 \mathrm{MHz}$, $\left.\mathrm{CDCl}_{3}\right) \partial / \mathrm{ppm}: 8.27\left(\mathrm{~s}, 4 \mathrm{H}, \mathrm{H}^{\mathrm{A} 3}\right), 7.60\left(\mathrm{~d}, J=8.5 \mathrm{~Hz}, 8 \mathrm{H}, \mathrm{H}^{\mathrm{B} 2}\right)$, $7.52\left(\mathrm{~s}, 4 \mathrm{H}, \mathrm{H}^{\mathrm{A} 5}\right), 7.14\left(\mathrm{~d}, J=8.7 \mathrm{~Hz}, 16 \mathrm{H}, \mathrm{H}^{\mathrm{C} 2}\right), 7.02(\mathrm{~d}, J=8.6$ $\left.\mathrm{Hz}, 8 \mathrm{H}, \mathrm{H}^{\mathrm{B} 3}\right), 6.89\left(\mathrm{~d}, J=8.4 \mathrm{~Hz}, 16 \mathrm{H}, \mathrm{H}^{\mathrm{C} 3}\right), 3.83\left(\mathrm{~s}, 24 \mathrm{H}, \mathrm{H}^{\mathrm{OMe}}\right)$, $2.47\left(\mathrm{~d}, J=7.4 \mathrm{~Hz}, 8 \mathrm{H}, \mathrm{H}^{\mathrm{a}}\right), 1.69\left(\mathrm{~m}, 4 \mathrm{H}, \mathrm{H}^{\mathrm{b}}\right), 0.54(\mathrm{~d}, J=6.7 \mathrm{~Hz}$, $\left.24 \mathrm{H}, \mathrm{H}^{\mathrm{c}}\right) .{ }^{13} \mathrm{C}$ NMR $\left(126 \mathrm{MHz}, \mathrm{CDCl}_{3}\right) \partial / \mathrm{ppm}: 160.5\left(\mathrm{C}^{\mathrm{A} 6}\right)$, 


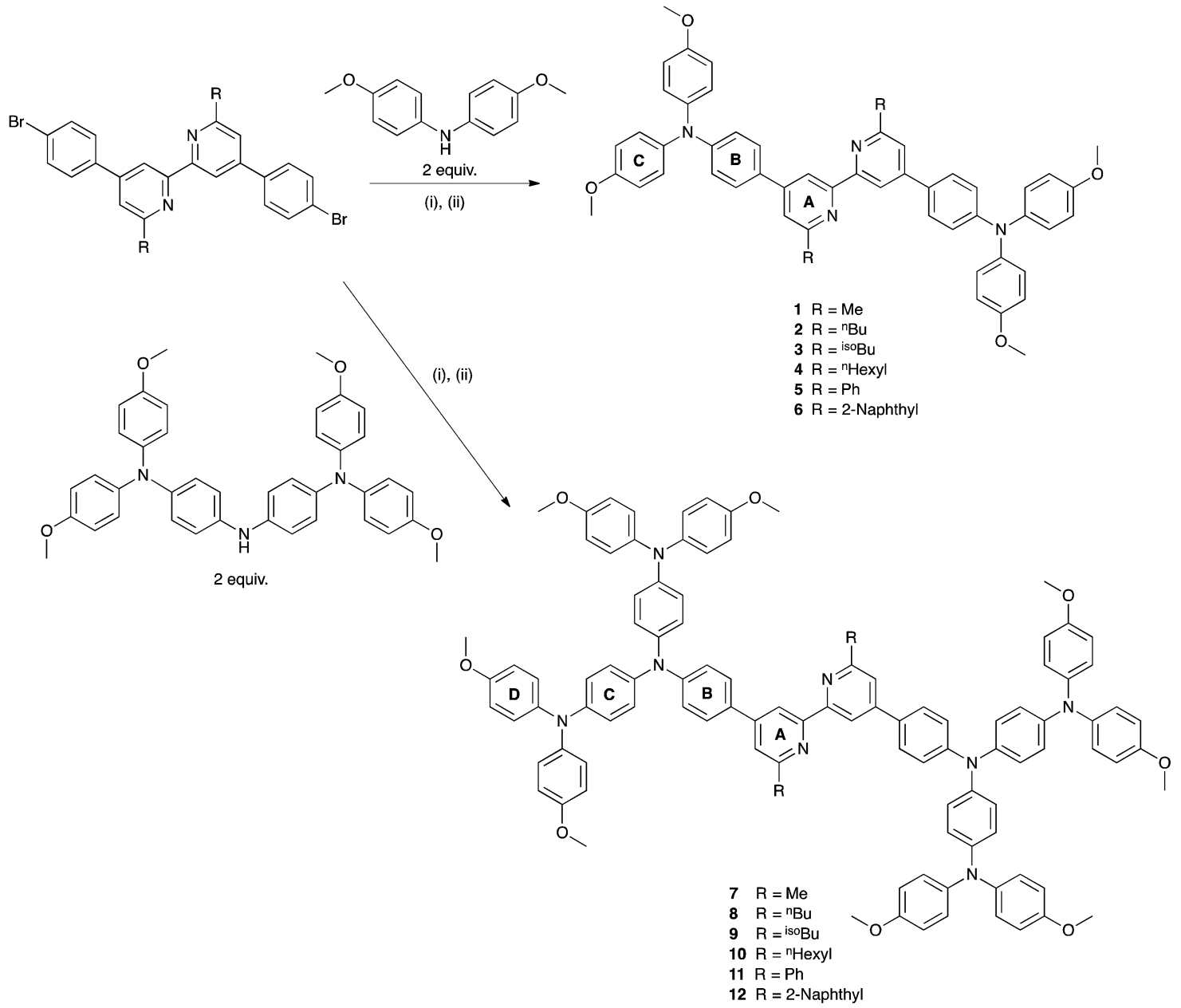

Scheme 2 Synthetic route to compounds 1-12. Conditions: (i) $\mathrm{NaO}{ }^{t} \mathrm{Bu}$; (ii) $\mathrm{Pd}(\mathrm{bda})_{2}, \mathrm{P}^{t} \mathrm{Bu}$, toluene, $100{ }^{\circ} \mathrm{C}, 16 \mathrm{~h}$. $\mathrm{Pd}(\mathrm{bda})_{2}=$ bis(dibenzylideneacetone)palladium(0)<smiles>Cc1cc(-c2ccc(P(O)O)cc2)cc(-c2cc(C)nc(-c3ccc([OH+][OH2+])cc3)c2)n1</smiles>

Scheme 3 Structure of anchoring ligand 13

156.9 $\left(\mathrm{C}^{\mathrm{C} 4}\right), 153.1\left(\mathrm{C}^{\mathrm{A} 2}\right), 149.9\left(\mathrm{C}^{\mathrm{B} 1}, \mathrm{C}^{\mathrm{B} 4}\right), 139.9\left(\mathrm{C}^{\mathrm{C} 1}\right), 127.9\left(\mathrm{C}^{\mathrm{B} 2}\right)$, $127.6\left(\mathrm{C}^{\mathrm{A} 4}, \mathrm{C}^{\mathrm{C} 2}\right), 122.8\left(\mathrm{C}^{\mathrm{A} 5}\right), 119.5\left(\mathrm{C}^{\mathrm{B} 3}\right), 116.5\left(\mathrm{C}^{\mathrm{A} 3}\right), 115.1\left(\mathrm{C}^{\mathrm{C} 3}\right)$, $55.7\left(\mathrm{C}^{\mathrm{OMe}}\right), 48.8\left(\mathrm{C}^{\mathrm{a}}\right), 28.5\left(\mathrm{C}^{\mathrm{b}}\right), 22.2\left(\mathrm{C}^{\mathrm{c}}\right) . \mathrm{IR}\left(\tilde{\nu} / \mathrm{cm}^{-1}\right): 3190(\mathrm{w})$, $3033(\mathrm{w}), 2997(\mathrm{w}), 2953(\mathrm{w}), 2929(\mathrm{w}), 2866(\mathrm{w}), 2834(\mathrm{w}), 1594$ (s), 1504 (s), 1463 (m), 1440 (m), 1322 (m), 1289 (m), 1239 (s), $1196(\mathrm{~m}), 1179(\mathrm{~m}), 1102(\mathrm{~m}), 1032(\mathrm{~m}), 825(\mathrm{~s}), 597(\mathrm{~m}), 574(\mathrm{~m})$, $557(\mathrm{~m}), 531$ (m). ESI MS (m/z): $1814.8\left[\mathrm{M}-\mathrm{PF}_{6}\right]^{+}$(calc. 1813.8), 875.6 $[3+\mathrm{H}]^{+}$(base peak, calc. 875.5). UV-VIS $\left(\mathrm{CH}_{2} \mathrm{Cl}_{2}, 1.0 \times\right.$ $\left.10^{-5} \mathrm{~mol} \mathrm{dm}{ }^{-3}\right): \lambda_{\max } / \mathrm{nm} 305\left(\varepsilon / \mathrm{dm}^{3} \mathrm{~mol}^{-1} \mathrm{~cm}^{-1} 63000\right), 393$ (58 700), 480 sh (28 200). Found: C, 70.14; H, 6.30; N, 5.48; $\mathrm{C}_{116} \mathrm{H}_{116} \mathrm{~N}_{8} \mathrm{O}_{8} \mathrm{CuPF}_{6} \cdot \mathrm{H}_{2} \mathrm{O}$ requires $\mathrm{C}, 70.48 ; \mathrm{H}, 6.02 ; \mathrm{N}, 5.67$ (\%).

\section{$\left[\mathrm{Cu}(4)_{2}\right]\left[\mathrm{PF}_{6}\right]$}

The method was as for $\left[\mathrm{Cu}(2)_{2}\right]\left[\mathrm{PF}_{6}\right]$ starting with $\left[\mathrm{Cu}(\mathrm{MeCN})_{4}\right]\left[\mathrm{PF}_{6}\right]$ (31.7 $\mathrm{mg}, 0.085 \mathrm{mmol}$ ) and 4 (158 $\mathrm{mg}, 0.17 \mathrm{mmol}) .\left[\mathrm{Cu}(4)_{2}\right]\left[\mathrm{PF}_{6}\right]$ was isolated as a red solid (153 $\mathrm{mg}, 0.074 \mathrm{mmol}, 86 \%) .{ }^{1} \mathrm{H} \mathrm{NMR}$ $\left(500 \mathrm{MHz}, \mathrm{CDCl}_{3}\right) \partial / \mathrm{ppm}: 8.26\left(\mathrm{~d}, J=1.4 \mathrm{~Hz}, 4 \mathrm{H}, \mathrm{H}^{\mathrm{A} 3}\right), 7.58(\mathrm{~d}$, $\left.J=8.9 \mathrm{~Hz}, 8 \mathrm{H}, \mathrm{H}^{\mathrm{B} 2}\right), 7.56\left(\mathrm{~d}, J=1.4 \mathrm{~Hz}, 4 \mathrm{H}, \mathrm{H}^{\mathrm{A} 5}\right), 7.13(\mathrm{~d}, J=9.0$ $\left.\mathrm{Hz}, 16 \mathrm{H}, \mathrm{H}^{\mathrm{C} 2}\right), 7.02\left(\mathrm{~d}, J=8.9 \mathrm{~Hz}, 8 \mathrm{H}, \mathrm{H}^{\mathrm{B} 3}\right), 6.89(\mathrm{~d}, J=9.0 \mathrm{~Hz}$, $\left.16 \mathrm{H}, \mathrm{H}^{\mathrm{C} 3}\right), 3.83\left(\mathrm{~s}, 24 \mathrm{H}, \mathrm{H}^{\mathrm{OMe}}\right), 2.61\left(\mathrm{t}, J=8.2 \mathrm{~Hz}, 8 \mathrm{H}, \mathrm{H}^{\mathrm{a}}\right), 1.38$ $\left(\mathrm{m}, 8 \mathrm{H}, \mathrm{H}^{\mathrm{b}}\right), 0.95\left(\mathrm{~m}, 8 \mathrm{H}, \mathrm{H}^{\mathrm{d}}\right), 0.86\left(\mathrm{~m}, 8 \mathrm{H}, \mathrm{H}^{\mathrm{c}}\right), 0.77\left(\mathrm{~m}, 8 \mathrm{H}, \mathrm{H}^{\mathrm{e}}\right)$, $0.62\left(\mathrm{t}, J=7.3 \mathrm{~Hz}, 12 \mathrm{H}, \mathrm{H}^{\mathrm{f}}\right) .{ }^{13} \mathrm{C} \mathrm{NMR}\left(126 \mathrm{MHz} \mathrm{CDCl}_{3}\right) \partial / \mathrm{ppm}$ : 161.6 $\left(\mathrm{C}^{\mathrm{A} 6}\right), 156.8\left(\mathrm{C}^{\mathrm{C} 4}\right), 152.6\left(\mathrm{C}^{\mathrm{A} 2}\right), 150.8\left(\mathrm{C}^{\mathrm{B} 4}\right), 150.2\left(\mathrm{C}^{\mathrm{B} 1}\right)$, $139.8\left(\mathrm{C}^{\mathrm{C} 1}\right), 127.8\left(\mathrm{C}^{\mathrm{B} 2}\right), 127.5\left(\mathrm{C}^{\mathrm{C} 2}\right), 127.2\left(\mathrm{C}^{\mathrm{A} 4}\right), 121.8\left(\mathrm{C}^{\mathrm{A} 5}\right)$, 119.4 $\left(\mathrm{C}^{\mathrm{B} 3}\right), 116.2\left(\mathrm{C}^{\mathrm{A} 3}\right), 115.1\left(\mathrm{C}^{\mathrm{C} 3}\right), 55.7\left(\mathrm{C}^{\mathrm{OMe}}\right), 40.1\left(\mathrm{C}^{\mathrm{a}}\right), 31.5$ $\left(\mathrm{C}^{\mathrm{e}}\right), 30.0\left(\mathrm{C}^{\mathrm{b}}\right), 29.5\left(\mathrm{C}^{\mathrm{c}}\right), 22.6\left(\mathrm{C}^{\mathrm{d}}\right), 14.1\left(\mathrm{C}^{\mathrm{f}}\right) . \mathrm{IR}\left(\tilde{\nu} / \mathrm{cm}^{-1}\right): 3187$ (w), $3036(\mathrm{w}), 2997(\mathrm{w}), 2947(\mathrm{w}), 2924(\mathrm{w}), 2853(\mathrm{w}), 2835(\mathrm{w})$, 1594 (s), 1504 (s), 1461 (m), 1327 (m), 1294 (m), 1239 (s), 1200 (m), $1179(\mathrm{~m}), 1102(\mathrm{~m}), 1031(\mathrm{~m}), 838(\mathrm{~s}), 825(\mathrm{~s}), 729(\mathrm{~m}), 663$ $(\mathrm{m}), 573(\mathrm{~m}), 557(\mathrm{~m}), 535(\mathrm{~m})$. ESI MS $(\mathrm{m} / \mathrm{z}): 1926.0\left[\mathrm{M}-\mathrm{PF}_{6}\right]^{+}$ (base peak, calc. 1924.5), 932.2 [4+ H] ${ }^{+}$(calc. 931.5). UV-VIS $\left(\mathrm{CH}_{2} \mathrm{Cl}_{2}, 1.0 \times 10^{-5} \mathrm{~mol} \mathrm{dm}{ }^{-3}\right): \lambda_{\max } / \mathrm{nm} 306\left(\varepsilon / \mathrm{dm}^{3} \mathrm{~mol}^{-1}\right.$ $\mathrm{cm}^{-1} 65$ 700), 388 (60 000), 480 sh (28 000). Found: C, 68.93; H, 
6.12; N, 5.46; $\mathrm{C}_{124} \mathrm{H}_{132} \mathrm{~N}_{8} \mathrm{O}_{8} \mathrm{CuPF}_{6} \cdot 4 \mathrm{H}_{2} \mathrm{O}$ requires $\mathrm{C}, 69.50 ; \mathrm{H}$, $6.58 ; \mathrm{N}, 5.23(\%)$.

\section{$\left[\mathrm{Cu}(5)_{2}\right]\left[\mathrm{PF}_{6}\right]$}

The method was as for $\left[\mathrm{Cu}(2)_{2}\right]\left[\mathrm{PF}_{6}\right]$ starting with $\left[\mathrm{Cu}(\mathrm{MeCN})_{4}\right]\left[\mathrm{PF}_{6}\right]$ (45.9 mg, $0.123 \mathrm{mmol}$ ) and 5 (225 mg, $0.246 \mathrm{mmol}$ ) in $\mathrm{CH}_{2} \mathrm{Cl}_{2}$ $(40 \mathrm{~mL})$, and the reaction mixture turned green-black. $\left[\mathrm{Cu}(5)_{2}\right]$ $\left[\mathrm{PF}_{6}\right]$ was isolated as a green-black solid $(225 \mathrm{mg}, 0.11 \mathrm{mmol}$, 90\%). ${ }^{1} \mathrm{H}$ NMR (500 MHz, $\mathrm{CDCl}_{3}$ ) d/ppm: 7.95 (d, J=1.2 Hz, 4H, $\left.\mathrm{H}^{\mathrm{A} 3}\right), 7.61\left(\mathrm{~d}, J=1.2 \mathrm{~Hz}, 4 \mathrm{H}, \mathrm{H}^{\mathrm{A} 5}\right), 7.58\left(\mathrm{~m}, 8 \mathrm{H}, \mathrm{H}^{\mathrm{D} 2}\right), 7.56(\mathrm{~d}, J=$ $\left.8.8 \mathrm{~Hz}, 8 \mathrm{H}, \mathrm{H}^{\mathrm{B} 2}\right), 7.17\left(\mathrm{~d}, J=9.0 \mathrm{~Hz}, 16 \mathrm{H}, \mathrm{H}^{\mathrm{C} 2}\right), 7.05(\mathrm{~d}, J=8.8$ $\left.\mathrm{Hz}, 8 \mathrm{H}, \mathrm{H}^{\mathrm{B} 3}\right), 7.03\left(\mathrm{~m}, 4 \mathrm{H}, \mathrm{H}^{\mathrm{D} 4}\right), 6.92\left(\mathrm{~d}, J=9.0 \mathrm{~Hz}, 16 \mathrm{H}, \mathrm{H}^{\mathrm{C} 3}\right)$, $7.03\left(\mathrm{~m}, 8 \mathrm{H}, \mathrm{H}^{\mathrm{D} 3}\right), 3.84\left(\mathrm{~s}, 24 \mathrm{H}, \mathrm{H}^{\mathrm{OMe}}\right) .{ }^{13} \mathrm{C} \mathrm{NMR}(126 \mathrm{MHz}$, $\left.\mathrm{CDCl}_{3}\right)$ d/ppm: $157.0\left(\mathrm{C}^{\mathrm{A} 6}\right), 156.9\left(\mathrm{C}^{\mathrm{C} 4}\right), 153.6\left(\mathrm{C}^{\mathrm{A} 2}\right), 150.9\left(\mathrm{C}^{\mathrm{B} 4}\right)$, $149.9\left(\mathrm{C}^{\mathrm{B} 1}\right), 139.8\left(\mathrm{C}^{\mathrm{C} 1}\right), 138.7\left(\mathrm{C}^{\mathrm{D} 1}\right), 129.2\left(\mathrm{C}^{\mathrm{D} 4}\right), 127.8\left(\mathrm{C}^{\mathrm{B} 2}\right)$, $127.7\left(\mathrm{C}^{\mathrm{D} 2}, \mathrm{C}^{\mathrm{D} 3}\right), 127.6\left(\mathrm{C}^{\mathrm{C} 2}\right), 127.2\left(\mathrm{C}^{\mathrm{A} 4}\right), 121.5\left(\mathrm{C}^{\mathrm{A} 5}\right), 119.4\left(\mathrm{C}^{\mathrm{B} 3}\right)$, $117.8\left(\mathrm{C}^{\mathrm{A} 3}\right), 115.1\left(\mathrm{C}^{\mathrm{C} 3}\right), 55.7\left(\mathrm{C}^{\mathrm{OMe}}\right)$. IR $\left(\tilde{\nu} / \mathrm{cm}^{-1}\right): 3036(\mathrm{w}), 2999$ (w), $2950(\mathrm{w}), 2931(\mathrm{w}), 2903(\mathrm{w}), 2832(\mathrm{w}), 1593(\mathrm{~s}), 1504(\mathrm{~s}), 1321$ (m), $1238(\mathrm{~s}), 1196(\mathrm{~m}), 1179(\mathrm{~m}), 1104(\mathrm{~m}), 1030(\mathrm{~m}), 824(\mathrm{~s}), 773$ (m), $741(\mathrm{~m}), 729(\mathrm{~m}), 696(\mathrm{~m}), 576(\mathrm{~m}), 557(\mathrm{~m}), 527(\mathrm{~m})$. ESI MS $(\mathrm{m} / \mathrm{z}): 1894.4\left[\mathrm{M}-\mathrm{PF}_{6}\right]^{+}$(calc. 1893.7), $915.9[5+\mathrm{H}]^{+}$(base peak, calc. 915.4). UV-VIS $\left(\mathrm{CH}_{2} \mathrm{Cl}_{2}, 1.0 \times 10^{-5} \mathrm{~mol} \mathrm{dm}^{-3}\right): \lambda_{\max } / \mathrm{nm}$ 286 ( $\left.\varepsilon / \mathrm{dm}^{3} \mathrm{~mol}^{-1} \mathrm{~cm}^{-1} 79300\right), 331$ (51 600), 401 (55 600), 560 sh (7000). Found: C, 73.04; H, 5.39; N, 5.30; $\mathrm{C}_{124} \mathrm{H}_{100} \mathrm{~N}_{8} \mathrm{O}_{8} \mathrm{CuPF}_{6}$ requires $\mathrm{C}, 73.05 ; \mathrm{H}, 4.94 ; \mathrm{N}, 5.50$ (\%).

\section{$\left[\mathbf{C u}(6)_{2}\right]\left[\mathrm{PF}_{6}\right]$}

The method was as for $\left[\mathrm{Cu}(2)_{2}\right]\left[\mathrm{PF}_{6}\right]$ starting with $\left[\mathrm{Cu}(\mathrm{MeCN})_{4}\right]\left[\mathrm{PF}_{6}\right]$ (27.6 mg, $0.074 \mathrm{mmol})$ and $6(150 \mathrm{mg}, 0.148 \mathrm{mmol})$ in $\mathrm{CH}_{2} \mathrm{Cl}_{2}$ $(40 \mathrm{~mL})$. The reaction mixture was green-black, and $\left[\mathrm{Cu}(6)_{2}\right]\left[\mathrm{PF}_{6}\right]$ was isolated as a green-black solid (153 mg, $0.068 \mathrm{mmol}, 92 \%)$. ${ }^{1} \mathrm{H}$ NMR (500 MHz, $\mathrm{CDCl}_{3}$ ) d/ppm: $8.22\left(\mathrm{~s}, 4 \mathrm{H}, \mathrm{H}^{\mathrm{D} 1}\right), 7.62(\mathrm{~m}, 4 \mathrm{H}$, $\left.\mathrm{H}^{\mathrm{D} 3}\right), 7.57\left(\mathrm{~m}, 8 \mathrm{H}, \mathrm{H}^{\mathrm{A} 5+\mathrm{D} 5 / \mathrm{D} 8}\right), 7.43\left(\mathrm{~d}, J=8.6 \mathrm{~Hz}, 4 \mathrm{H}, \mathrm{H}^{\mathrm{D} 4}\right), 7.32(\mathrm{~m}$, $\left.16 \mathrm{H}, \mathrm{H}^{\mathrm{A} 3+\mathrm{B} 2+\mathrm{D} 6 / \mathrm{D} 7}\right), 7.20\left(\mathrm{~d}, J=8.9 \mathrm{~Hz}, 16 \mathrm{H}, \mathrm{H}^{\mathrm{C} 2}\right), 7.14(\mathrm{~m}, 8 \mathrm{H}$, $\left.\mathrm{H}^{\mathrm{D} 5 / \mathrm{D} 8+\mathrm{D} 6 / \mathrm{D} 7}\right), 7.03\left(\mathrm{~d}, J=8.7 \mathrm{~Hz}, 8 \mathrm{H}, \mathrm{H}^{\mathrm{B} 3}\right), 6.95(\mathrm{~d}, J=9.0 \mathrm{~Hz}, 16 \mathrm{H}$, $\mathrm{H}^{\mathrm{C} 3}$ ), 3.86 (s, 24H, $\left.\mathrm{H}^{\mathrm{OMe}}\right) .{ }^{13} \mathrm{C}$ NMR (126 $\left.\mathrm{MHz} \mathrm{CDCl}_{3}\right) \mathrm{d} / \mathrm{ppm}$ : $156.9\left(\mathrm{C}^{\mathrm{C} 4}\right), 156.5\left(\mathrm{C}^{\mathrm{A} 6}\right), 153.5\left(\mathrm{C}^{\mathrm{A} 2}\right), 150.8\left(\mathrm{C}^{\mathrm{B} 4}\right), 149.8\left(\mathrm{C}^{\mathrm{B} 1}\right), 139.9$ $\left(\mathrm{C}^{\mathrm{C} 1}\right), 136.0\left(\mathrm{C}^{\mathrm{D} 2}\right), 133.3\left(\mathrm{C}^{\mathrm{D} 4 \mathrm{a} / \mathrm{D} 8 \mathrm{a}}\right), 132.2\left(\mathrm{C}^{\mathrm{D} 4 \mathrm{a} / \mathrm{D} 8 \mathrm{a}}\right), 128.1$ $\left(\mathrm{C}^{\mathrm{D} 5 / \mathrm{D} 6 / \mathrm{D} 7 / \mathrm{D} 8}\right), 127.9\left(\mathrm{C}^{\mathrm{B} 2}\right), 127.6\left(\mathrm{C}^{\mathrm{C} 2}\right), 127.5\left(\mathrm{C}^{\mathrm{D} 1+\mathrm{D} 4+\mathrm{D} 5 / \mathrm{D} 8}\right)$, $127.2\left(\mathrm{C}^{\mathrm{A} 4}\right), 127.1\left(\mathrm{C}^{\mathrm{D} 6 / \mathrm{D} 7}\right), 126.4\left(\mathrm{C}^{\mathrm{D} 5 / \mathrm{D} 6 / \mathrm{D} 7 / \mathrm{D} 8}\right), 125.1\left(\mathrm{C}^{\mathrm{D} 3}\right)$, 121.2 $\left(\mathrm{C}^{\mathrm{A} 5}\right), 119.2\left(\mathrm{C}^{\mathrm{B} 3}\right), 117.4\left(\mathrm{C}^{\mathrm{A} 3}\right), 115.2\left(\mathrm{C}^{\mathrm{C} 3}\right), 55.7\left(\mathrm{C}^{\mathrm{OMe}}\right) . \mathrm{IR}$ $\left(\tilde{\nu} / \mathrm{cm}^{-1}\right): 3038(\mathrm{w}), 2999(\mathrm{w}), 2950(\mathrm{w}), 2929(\mathrm{w}), 2905(\mathrm{w}), 2832$ (w), 1592 (s), 1504 (s), 1320 (m), 1239 (m), 1196 (m), 1179 (m), $1102(\mathrm{~m}), 1032(\mathrm{~m}), 824(\mathrm{~s}), 781(\mathrm{~m}), 755(\mathrm{~m}), 741(\mathrm{~m}), 576(\mathrm{~m})$, $557(\mathrm{~m}), 533(\mathrm{~m}), 477(\mathrm{~m})$. ESI MS $(\mathrm{m} / \mathrm{z}): 2094.8\left[\mathrm{M}-\mathrm{PF}_{6}\right]^{+}$(base peak, calc. 2093.8.), $1016.1[6+\mathrm{H}]^{+}$(calc. 1015.4). UV-VIS $\left(\mathrm{CH}_{2} \mathrm{Cl}_{2}, 1.0 \times 10^{-5} \mathrm{~mol} \mathrm{dm}{ }^{-3}\right): \lambda_{\max } / \mathrm{nm} 290\left(\varepsilon / \mathrm{dm}^{3} \mathrm{~mol}^{-1}\right.$ $\mathrm{cm}^{-1} 103$ 100), $340 \mathrm{sh}$ (55 400), 408 (58 000), 576 (5900). Found: C, 73.11; H, 5.18; N, 4.98; $\mathrm{C}_{140} \mathrm{H}_{108} \mathrm{~N}_{8} \mathrm{O}_{8} \mathrm{CuPF}_{6} \cdot 3 \mathrm{H}_{2} \mathrm{O}$ requires C, 73.33; H, 5.01; N, 4.89 (\%).

\section{$\left[\mathrm{Cu}(8)_{2}\right]\left[\mathrm{PF}_{6}\right]$}

The method was as for $\left[\mathrm{Cu}(2)_{2}\right]\left[\mathrm{PF}_{6}\right]$ starting with $\left[\mathrm{Cu}(\mathrm{MeCN})_{4}\right]\left[\mathrm{PF}_{6}\right]$ (27.6 $\mathrm{mg}, 73.9 \mu \mathrm{mol})$ and $8(246 \mathrm{mg}, 0.148 \mathrm{mmol})$ in $\mathrm{MeCN}$ $(18 \mathrm{~mL})$ and $\mathrm{CH}_{2} \mathrm{Cl}_{2}(18 \mathrm{~mL}) \cdot\left[\mathrm{Cu}(8)_{2}\right]\left[\mathrm{PF}_{6}\right]$ was isolated as a red solid (202 mg, $57.2 \mu \mathrm{mol}, 77 \%) .{ }^{1} \mathrm{H} \mathrm{NMR}\left(500 \mathrm{MHz}, \mathrm{CD}_{2} \mathrm{Cl}_{2}\right.$ ) d/ppm: $8.34\left(\mathrm{~d}, J=1.0 \mathrm{~Hz}, 4 \mathrm{H}, \mathrm{H}^{\mathrm{A} 3}\right), 7.65\left(\mathrm{~d}, J=8.8 \mathrm{~Hz}, 8 \mathrm{H}, \mathrm{H}^{\mathrm{B} 2}\right)$, $7.60\left(\mathrm{~d}, J=1.0 \mathrm{~Hz}, 4 \mathrm{H}, \mathrm{H}^{\mathrm{A} 5}\right), 7.11\left(\mathrm{~d}, J=8.8 \mathrm{~Hz}, 8 \mathrm{H}, \mathrm{H}^{\mathrm{B} 3}\right), 7.07$ $\left(\mathrm{d}, J=9.0 \mathrm{~Hz}, 32 \mathrm{H}, \mathrm{H}^{\mathrm{D} 2}\right), 7.02\left(\mathrm{~d}, J=8.9 \mathrm{~Hz}, 16 \mathrm{H}, \mathrm{H}^{\mathrm{C} 2}\right), 6.90$ $\left(\mathrm{d}, J=8.9 \mathrm{~Hz}, 16 \mathrm{H}, \mathrm{H}^{\mathrm{C} 3}\right), 6.84\left(\mathrm{~d}, J=9.0 \mathrm{~Hz}, 32 \mathrm{H}, \mathrm{H}^{\mathrm{D} 3}\right), 3.77$ $\left(\mathrm{s}, 48 \mathrm{H}, \mathrm{H}^{\mathrm{OMe}}\right), 2.64\left(\mathrm{t}, J=8.1 \mathrm{~Hz}, 8 \mathrm{H}, \mathrm{H}^{\mathrm{a}}\right), 1.39\left(\mathrm{~m}, 8 \mathrm{H}, \mathrm{H}^{\mathrm{b}}\right), 0.90$ $\left(\mathrm{m}, 8 \mathrm{H}, \mathrm{H}^{\mathrm{c}}\right), 0.48\left(\mathrm{t}, J=7.3 \mathrm{~Hz}, 12 \mathrm{H}, \mathrm{H}^{\mathrm{d}}\right) .{ }^{13} \mathrm{C} \mathrm{NMR}(126 \mathrm{MHz}$, $\left.\mathrm{CD}_{2} \mathrm{Cl}_{2}\right) \partial /$ ppm: $162.0\left(\mathrm{C}^{\mathrm{A} 6}\right), 156.5\left(\mathrm{C}^{\mathrm{D} 4}\right), 153.1\left(\mathrm{C}^{\mathrm{A} 2}\right), 151.0\left(\mathrm{C}^{\mathrm{B} 4}\right)$, $150.5\left(\mathrm{C}^{\mathrm{A} 4}\right), 146.2\left(\mathrm{C}^{\mathrm{C} 4}\right), 141.4\left(\mathrm{C}^{\mathrm{D} 1}\right), 139.9\left(\mathrm{C}^{\mathrm{C} 1}\right), 128.2\left(\mathrm{C}^{\mathrm{B} 2}\right)$, $128.0\left(\mathrm{C}^{\mathrm{B} 1}\right), 127.2\left(\mathrm{C}^{\mathrm{C} 2}\right), 126.9\left(\mathrm{C}^{\mathrm{D} 2}\right), 122.1\left(\mathrm{C}^{\mathrm{A} 5+\mathrm{C} 3}\right), 120.1\left(\mathrm{C}^{\mathrm{B} 3}\right)$, $116.8\left(\mathrm{C}^{\mathrm{A} 3}\right), 115.2\left(\mathrm{C}^{\mathrm{D} 3}\right), 56.0\left(\mathrm{C}^{\mathrm{OMe}}\right), 40.2\left(\mathrm{C}^{\mathrm{a}}\right), 32.4\left(\mathrm{C}^{\mathrm{b}}\right), 23.1$ $\left(\mathrm{C}^{\mathrm{c}}\right), 13.8\left(\mathrm{C}^{\mathrm{d}}\right)$. IR $\left(\tilde{\nu} / \mathrm{cm}^{-1}\right): 3036(\mathrm{w}), 2997(\mathrm{w}), 2954(\mathrm{w}), 2929(\mathrm{w})$, 2905 (w), 2834 (w), 1595 (m), 1497 (s), 1313 (m), 1237 (s), 1033 (m), $824(\mathrm{~s}), 576(\mathrm{~m}), 539(\mathrm{~m})$. MALDI-TOF MS (m/z): 1728.2 $[\mathrm{Cu}(8)]^{+}$(calc. 1726.7), $1664.8[8+\mathrm{H}]^{+}$(calc. 1664.8). UV-VIS $\left(\mathrm{CH}_{2} \mathrm{Cl}_{2}, 1.0 \times 10^{-5} \mathrm{~mol} \mathrm{dm}{ }^{-3}\right): \lambda_{\max } / \mathrm{nm} 225\left(\varepsilon / \mathrm{dm}^{3} \mathrm{~mol}^{-1}\right.$ $\mathrm{cm}^{-1} 170$ 200), 309 (159 600), $340 \mathrm{sh}$ (134 200), $480 \mathrm{sh}$ (39 600). Found: C, 73.53; H, 6.33; N, 6.03; $\mathrm{C}_{220} \mathrm{H}_{204} \mathrm{CuF}_{6} \mathrm{~N}_{16} \mathrm{O}_{16} \mathrm{P} \cdot 3 \mathrm{H}_{2} \mathrm{O}$ requires $\mathrm{C}, 73.59 ; \mathrm{H}, 5.89 ; \mathrm{N}, 6.24(\%)$.

\section{$\left[\mathrm{Cu}(9)_{2}\right]\left[\mathrm{PF}_{6}\right]$}

The method was as for $\left[\mathrm{Cu}(2)_{2}\right]\left[\mathrm{PF}_{6}\right]$ starting with $\left[\mathrm{Cu}(\mathrm{MeCN})_{4}\right]\left[\mathrm{PF}_{6}\right]$ $(31.9 \mathrm{mg}, 85.7 \mu \mathrm{mol})$ and $\mathbf{9}(285 \mathrm{mg}, 171 \mu \mathrm{mol})$ in a mixture of MeCN $(10 \mathrm{~mL})$ and $\mathrm{CH}_{2} \mathrm{Cl}_{2}(20 \mathrm{~mL})$. $\left[\mathrm{Cu}(9)_{2}\right]\left[\mathrm{PF}_{6}\right]$ was isolated as red solid (251 mg, $70.9 \mu \mathrm{mol}, 83 \%) .{ }^{1} \mathrm{H} \mathrm{NMR}\left(500 \mathrm{MHz}, \mathrm{CD}_{2} \mathrm{Cl}_{2}\right.$ ) d/ppm: $8.35\left(\mathrm{~d}, J=1.3 \mathrm{~Hz}, 4 \mathrm{H}, \mathrm{H}^{\mathrm{A} 3}\right), 7.66\left(\mathrm{~d}, J=8.9 \mathrm{~Hz}, 8 \mathrm{H}, \mathrm{H}^{\mathrm{B} 2}\right)$, $7.56\left(\mathrm{~d}, J=1.3 \mathrm{~Hz}, 4 \mathrm{H}, \mathrm{H}^{\mathrm{A} 5}\right), 7.11\left(\mathrm{~d}, J=8.8 \mathrm{~Hz}, 8 \mathrm{H}, \mathrm{H}^{\mathrm{B} 3}\right), 7.07(\mathrm{~d}$, $\left.J=9.0 \mathrm{~Hz}, 32 \mathrm{H}, \mathrm{H}^{\mathrm{D} 2}\right), 7.02\left(\mathrm{~d}, J=9.0 \mathrm{~Hz}, 16 \mathrm{H}, \mathrm{H}^{\mathrm{C} 2}\right), 6.89(\mathrm{~d}, J=$ $\left.9.0 \mathrm{~Hz}, 16 \mathrm{H}, \mathrm{H}^{\mathrm{C} 3}\right), 6.84\left(\mathrm{~d}, J=9.0 \mathrm{~Hz}, 32 \mathrm{H}, \mathrm{H}^{\mathrm{D} 3}\right), 3.77(\mathrm{~s}, 48 \mathrm{H}$, $\left.\mathrm{H}^{\mathrm{OMe}}\right), 2.49\left(\mathrm{~d}, J=7.2 \mathrm{~Hz}, 8 \mathrm{H}, \mathrm{H}^{\mathrm{a}}\right), 1.72\left(\mathrm{~m}, 4 \mathrm{H}, \mathrm{H}^{\mathrm{b}}\right), 0.54(\mathrm{~d}, J=$ $\left.6.6 \mathrm{~Hz}, 24 \mathrm{H}, \mathrm{H}^{\mathrm{c}}\right) .{ }^{13} \mathrm{C}$ NMR (126 MHz, $\left.\mathrm{CD}_{2} \mathrm{Cl}_{2}\right)$ d/ppm: 160.9 $\left(\mathrm{C}^{\mathrm{A} 6}\right), 156.5\left(\mathrm{C}^{\mathrm{D} 4}\right), 153.6\left(\mathrm{C}^{\mathrm{A} 2}\right), 151.1\left(\mathrm{C}^{\mathrm{B} 4}\right), 150.2\left(\mathrm{C}^{\mathrm{A} 4}\right), 146.2$ $\left(\mathrm{C}^{\mathrm{C} 4}\right), 141.5\left(\mathrm{C}^{\mathrm{D} 1}\right), 139.9\left(\mathrm{C}^{\mathrm{C} 1}\right), 128.2\left(\mathrm{C}^{\mathrm{B} 2}\right), 127.8\left(\mathrm{C}^{\mathrm{B} 1}\right), 127.2$ $\left(\mathrm{C}^{\mathrm{C} 2}\right), 126.9\left(\mathrm{C}^{\mathrm{D} 2}\right), 123.2\left(\mathrm{C}^{\mathrm{A} 5}\right), 122.1\left(\mathrm{C}^{\mathrm{C} 3}\right), 120.2\left(\mathrm{C}^{\mathrm{B} 3}\right), 117.0$ $\left(\mathrm{C}^{\mathrm{A} 3}\right), 115.2\left(\mathrm{C}^{\mathrm{D} 3}\right), 56.0\left(\mathrm{C}^{\mathrm{OMe}}\right), 49.2\left(\mathrm{C}^{\mathrm{a}}\right), 28.8\left(\mathrm{C}^{\mathrm{b}}\right), 22.4\left(\mathrm{C}^{\mathrm{c}}\right) . \mathrm{IR}$ $\left(\tilde{\nu} / \mathrm{cm}^{-1}\right): 3030(\mathrm{w}), 2997(\mathrm{w}), 2970(\mathrm{w}), 2952(\mathrm{w}), 2926(\mathrm{w}), 2903$ (w), $2866(\mathrm{w}), 2834(\mathrm{w}), 1595(\mathrm{~m}), 1497$ (s), $1312(\mathrm{~m}), 1236$ (s), $1034(\mathrm{~m}), 824(\mathrm{~s}), 576(\mathrm{~m}), 528(\mathrm{~m})$. MALDI-TOF MS (m/z): 3397.1 $\left[\mathrm{M}-\mathrm{PF}_{6}\right]^{+}$(calc. 3391.5), $1729.1[\mathrm{Cu}(9)]^{+}$(calc. 1726.7), 1666.0 $[9+\mathrm{H}]^{+}$(calc. 1664.8). UV-VIS $\left(\mathrm{CH}_{2} \mathrm{Cl}_{2}, 1.0 \times 10^{-5} \mathrm{~mol} \mathrm{dm}^{-3}\right)$ : $\lambda_{\max } / \mathrm{nm} 225\left(\varepsilon / \mathrm{dm}^{3} \mathrm{~mol}^{-1} \mathrm{~cm}^{-1} 164000\right), 309$ (159 900), $340 \mathrm{sh}$ (134 000), 480 sh (41 400). Found: C, 74.30; H, 6.03; N, 6.49; $\mathrm{C}_{220} \mathrm{H}_{204} \mathrm{~N}_{16} \mathrm{O}_{16} \mathrm{CuPF}_{6}$ requires C, 74.71; H, 5.81; N, 6.34 (\%).

\section{$\left[\mathrm{Cu}(10)_{2}\right]\left[\mathrm{PF}_{6}\right]$}

The method was as for $\left[\mathrm{Cu}(2)_{2}\right]\left[\mathrm{PF}_{6}\right]$ starting with $\left[\mathrm{Cu}(\mathrm{MeCN})_{4}\right]\left[\mathrm{PF}_{6}\right]$ $(29.5 \mathrm{mg}, 79.1 \mu \mathrm{mol})$ and $10(272 \mathrm{mg}, 158 \mu \mathrm{mol})$ in a mixture of MeCN $(20 \mathrm{~mL})$ and $\mathrm{CH}_{2} \mathrm{Cl}_{2}(20 \mathrm{~mL}) \cdot\left[\mathrm{Cu}(\mathbf{1 0})_{2}\right]\left[\mathrm{PF}_{6}\right]$ was isolated as red solid (242 mg, 66.2 $\mu \mathrm{mol}, 84 \%) .{ }^{1} \mathrm{H}$ NMR $(500 \mathrm{MHz}$, $\left.\mathrm{CD}_{2} \mathrm{Cl}_{2}\right) \partial / \mathrm{ppm}: 8.34\left(\mathrm{~d}, J=1.2 \mathrm{~Hz}, 4 \mathrm{H}, \mathrm{H}^{\mathrm{A} 3}\right), 7.65(\mathrm{~d}, J=8.9 \mathrm{~Hz}$, $\left.8 \mathrm{H}, \mathrm{H}^{\mathrm{B} 2}\right), 7.61\left(\mathrm{~d}, J=1.2 \mathrm{~Hz}, 4 \mathrm{H}, \mathrm{H}^{\mathrm{A} 5}\right), 7.11(\mathrm{~d}, J=8.8 \mathrm{~Hz}, 8 \mathrm{H}$, $\left.\mathrm{H}^{\mathrm{B} 3}\right), 7.07\left(\mathrm{~d}, J=9.0 \mathrm{~Hz}, 32 \mathrm{H}, \mathrm{H}^{\mathrm{D} 2}\right), 7.02(\mathrm{~d}, J=9.0 \mathrm{~Hz}, 16 \mathrm{H}$, $\left.\mathrm{H}^{\mathrm{C} 2}\right), 6.90\left(\mathrm{~d}, J=9.0 \mathrm{~Hz}, 16 \mathrm{H}, \mathrm{H}^{\mathrm{C} 3}\right), 6.84(\mathrm{~d}, J=9.0 \mathrm{~Hz}, 32 \mathrm{H}$, $\left.\mathrm{H}^{\mathrm{D} 3}\right), 3.77\left(\mathrm{~s}, 48 \mathrm{H}, \mathrm{H}^{\mathrm{OMe}}\right), 2.63\left(\mathrm{t}, J=8.2 \mathrm{~Hz}, 8 \mathrm{H}, \mathrm{H}^{\mathrm{a}}\right), 1.40(\mathrm{~m}$, $\left.4 \mathrm{H}, \mathrm{H}^{\mathrm{b}}\right), 0.95\left(\mathrm{~m}, 8 \mathrm{H}, \mathrm{H}^{\mathrm{e}}\right), 0.87\left(\mathrm{~m}, 8 \mathrm{H}, \mathrm{H}^{\mathrm{c}}\right), 0.79\left(\mathrm{~m}, 8 \mathrm{H}, \mathrm{H}^{\mathrm{d}}\right)$, $0.61\left(\mathrm{t}, J=7.3 \mathrm{~Hz}, 12 \mathrm{H}, \mathrm{H}^{\mathrm{f}}\right) .{ }^{13} \mathrm{C} \mathrm{NMR}\left(126 \mathrm{MHz}, \mathrm{CD}_{2} \mathrm{Cl}_{2}\right) \partial / \mathrm{ppm}$ : $162.1\left(\mathrm{C}^{\mathrm{A} 6}\right), 156.5\left(\mathrm{C}^{\mathrm{D} 4}\right), 153.1\left(\mathrm{C}^{\mathrm{A} 2}\right), 151.0\left(\mathrm{C}^{\mathrm{B} 4}\right), 150.5\left(\mathrm{C}^{\mathrm{A} 4}\right)$, 
146.2 $\left(\mathrm{C}^{\mathrm{C} 4}\right), 141.4\left(\mathrm{C}^{\mathrm{D} 1}\right), 139.9\left(\mathrm{C}^{\mathrm{C} 1}\right), 128.2\left(\mathrm{C}^{\mathrm{B} 2}\right), 127.9\left(\mathrm{C}^{\mathrm{B} 1}\right)$, $127.1\left(\mathrm{C}^{\mathrm{C} 2}\right), 126.9\left(\mathrm{C}^{\mathrm{D} 2}\right), 122.2\left(\mathrm{C}^{\mathrm{A} 5}\right), 122.1\left(\mathrm{C}^{\mathrm{C} 3}\right), 120.2\left(\mathrm{C}^{\mathrm{B} 3}\right)$, 116.6 $\left(\mathrm{C}^{\mathrm{A} 3}\right), 115.2\left(\mathrm{C}^{\mathrm{D} 3}\right), 56.0\left(\mathrm{C}^{\mathrm{OMe}}\right), 40.6\left(\mathrm{C}^{\mathrm{a}}\right), 32.0\left(\mathrm{C}^{\mathrm{d}}\right), 30.5$ $\left(\mathrm{C}^{\mathrm{b}}\right), 29.9\left(\mathrm{C}^{\mathrm{c}}\right), 23.0\left(\mathrm{C}^{\mathrm{e}}\right), 14.3\left(\mathrm{C}^{\mathrm{f}}\right) . \operatorname{IR}\left(\tilde{\nu} / \mathrm{cm}^{-1}\right): 3037(\mathrm{w}), 2997(\mathrm{w})$, 2950 (w), 2928 (w), 2853 (w), 2834 (w), 1594 (m), 1495 (s), 1312 (m), 1235 (s), $1032(\mathrm{~m}), 822(\mathrm{~s}), 575(\mathrm{~m}), 536(\mathrm{~m})$. MALDI-TOF MS $(\mathrm{m} / \mathrm{z}): 1784.7[\mathrm{Cu}(\mathbf{1 0})]^{+}$(calc. 1782.8), $1720.9[\mathbf{1 0}+\mathrm{H}]^{+}$(calc. 1720.9). UV-VIS $\left(\mathrm{CH}_{2} \mathrm{Cl}_{2}, 1.0 \times 10^{-5} \mathrm{~mol} \mathrm{dm}{ }^{-3}\right): \lambda_{\max } / \mathrm{nm} 225$ ( $\left.\varepsilon / \mathrm{dm}^{3} \mathrm{~mol}^{-1} \mathrm{~cm}^{-1} 164100\right), 309$ (155 400), 340 sh (131 200), 480 sh (42 200). Found: C, 73.54; H, 6.25; N, 6.14; $\mathrm{C}_{228} \mathrm{H}_{220^{-}}$ $\mathrm{N}_{16} \mathrm{O}_{16} \mathrm{CuPF}_{6} \cdot 4 \mathrm{H}_{2} \mathrm{O}$ requires $\mathrm{C}, 73.60 ; \mathrm{H}, 6.18 ; \mathrm{N}, 6.02(\%)$.

\section{$\left[\mathrm{Cu}(11)_{2}\right]\left[\mathrm{PF}_{6}\right]$}

The method was as for $\left[\mathrm{Cu}(2)_{2}\right]\left[\mathrm{PF}_{6}\right]$ starting with $\left[\mathrm{Cu}(\mathrm{MeCN})_{4}\right]\left[\mathrm{PF}_{6}\right]$ $(27.8 \mathrm{mg}, 74.5 \mu \mathrm{mol})$ and $11(254 \mathrm{mg}, 149 \mu \mathrm{mol})$ in $\mathrm{CH}_{2} \mathrm{Cl}_{2}$ $(40 \mathrm{~mL})$; the reaction mixture turned green-black. $\left[\mathrm{Cu}(\mathbf{1 1})_{2}\right]\left[\mathrm{PF}_{6}\right]$ was isolated as a green-black solid $(233 \mathrm{mg}, 64.3 \mu \mathrm{mol}, 86 \%) .{ }^{1} \mathrm{H}$ NMR (500 MHz, $\mathrm{CD}_{2} \mathrm{Cl}_{2}$ ) d/ppm: $8.02\left(\mathrm{~d}, J=1.7 \mathrm{~Hz}, 4 \mathrm{H}, \mathrm{H}^{\mathrm{A} 3}\right)$, $7.66\left(\mathrm{~d}, J=1.7 \mathrm{~Hz}, 4 \mathrm{H}, \mathrm{H}^{\mathrm{A} 5}\right), 7.63\left(\mathrm{~m}, 16 \mathrm{H}, \mathrm{H}^{\mathrm{B} 2+\mathrm{E} 2}\right), 7.14(\mathrm{~d}, J=$ $\left.8.5 \mathrm{~Hz}, 8 \mathrm{H}, \mathrm{H}^{\mathrm{B} 3}\right), 7.09\left(\mathrm{~d}, J=9.0 \mathrm{~Hz}, 32 \mathrm{H}, \mathrm{H}^{\mathrm{D} 2}\right), 7.05(\mathrm{~m}, 20 \mathrm{H}$, $\left.\mathrm{H}^{\mathrm{C} 2+\mathrm{E} 4}\right), 6.92\left(\mathrm{~m}, 24 \mathrm{H}, \mathrm{H}^{\mathrm{C} 3+\mathrm{E} 3}\right), 6.86\left(\mathrm{~d}, J=9.0 \mathrm{~Hz}, 32 \mathrm{H}, \mathrm{H}^{\mathrm{D} 3}\right)$, 3.78 (s, 48H, H $\left.{ }^{\mathrm{OMe}}\right) .{ }^{13} \mathrm{C}$ NMR (126 MHz, $\mathrm{CD}_{2} \mathrm{Cl}_{2}$ ) $\mathrm{d} / \mathrm{ppm}: 157.4$ $\left(\mathrm{C}^{\mathrm{A} 6}\right), 156.5\left(\mathrm{C}^{\mathrm{D} 4}\right), 154.2\left(\mathrm{C}^{\mathrm{A} 2}\right), 151.1\left(\mathrm{C}^{\mathrm{B} 4}\right), 150.2\left(\mathrm{C}^{\mathrm{A} 4}\right), 146.6$ $\left(\mathrm{C}^{\mathrm{C} 4}\right), 141.5\left(\mathrm{C}^{\mathrm{D} 1}\right), 139.8\left(\mathrm{C}^{\mathrm{C} 1}\right), 139.2\left(\mathrm{C}^{\mathrm{E} 1}\right), 129.5\left(\mathrm{C}^{\mathrm{E} 4}\right), 128.2$ $\left(\mathrm{C}^{\mathrm{E} 2}\right), 128.0\left(\mathrm{C}^{\mathrm{E} 3}\right), 127.9\left(\mathrm{C}^{\mathrm{B} 1}\right), 127.2\left(\mathrm{C}^{\mathrm{C} 1}\right), 126.9\left(\mathrm{C}^{\mathrm{D} 2}\right), 122.1$ $\left(\mathrm{C}^{\mathrm{C} 3}\right), 121.9\left(\mathrm{C}^{\mathrm{A} 5}\right), 120.1\left(\mathrm{C}^{\mathrm{B} 3}\right), 118.4\left(\mathrm{C}^{\mathrm{A} 3}\right), 115.2\left(\mathrm{C}^{\mathrm{D} 3}\right), 55.9$ $\left(\mathrm{C}^{\mathrm{OMe}}\right)$. IR ( $\left.\tilde{\nu} / \mathrm{cm}^{-1}\right): 3033(\mathrm{w}), 3004(\mathrm{w}), 2989(\mathrm{w}), 2952(\mathrm{w}), 2929$ (w), 2903 (w), 2834 (w), 1593 (m), 1497 (s), 1314 (m), 1238 (s), 1032 (m), 824 (s), 765 (m), 750 (m), 576 (m), 538 (m). MALDITOF MS $(\mathrm{m} / \mathrm{z}): 3478.9\left[\mathrm{M}-\mathrm{PF}_{6}\right]^{+}$(calc. 3471.4), 1769.1 $[\mathrm{Cu}(\mathbf{1 1})]^{+}$(calc. 1766.7), $1705.7[\mathbf{1 1}+\mathrm{H}]^{+}$(calc. 1704.7). UV-VIS $\left(\mathrm{CH}_{2} \mathrm{Cl}_{2}, 1.0 \times 10^{-5} \mathrm{~mol} \mathrm{dm}{ }^{-3}\right): \lambda_{\max } / \mathrm{nm} 226\left(\varepsilon / \mathrm{dm}^{3} \mathrm{~mol}^{-1}\right.$ $\mathrm{cm}^{-1} 225$ 900), 308 (190 100), $340 \mathrm{sh}$ (166 000), $435 \mathrm{sh}$ (57 000), 580 sh (11 500). Found: C, 73.04; H, 5.16; N, 6.16; $\mathrm{C}_{228} \mathrm{H}_{188^{-}}$ $\mathrm{N}_{16} \mathrm{O}_{16} \mathrm{CuPF}_{6} \cdot 2 \mathrm{CH}_{2} \mathrm{Cl}_{2}$ requires $\mathrm{C}, 72.96 ; \mathrm{H}, 5.11 ; \mathrm{N}, 5.92(\%)$.

\section{$\left[\mathrm{Cu}(12)_{2}\right]\left[\mathrm{PF}_{6}\right]$}

The method was as for $\left[\mathrm{Cu}(2)_{2}\right]\left[\mathrm{PF}_{6}\right]$ starting with $\left[\mathrm{Cu}(\mathrm{MeCN})_{4}\right]\left[\mathrm{PF}_{6}\right]$ $(25.3 \mathrm{mg}, 67.8 \mu \mathrm{mol})$ and $12(245 \mathrm{mg}, 136 \mu \mathrm{mol})$ in $\mathrm{CH}_{2} \mathrm{Cl}_{2}$ $(100 \mathrm{~mL})$; the reaction mixture was green-black. $\left[\mathrm{Cu}(12)_{2}\right]\left[\mathrm{PF}_{6}\right]$ was isolated as green-black solid (231 mg, $60.6 \mu \mathrm{mol}, 89 \%) .{ }^{1} \mathrm{H}$ NMR (500 MHz, $\mathrm{CD}_{2} \mathrm{Cl}_{2}$ ) $\partial / \mathrm{ppm}: 8.29$ (d, $J=1.4 \mathrm{~Hz}, 4 \mathrm{H}, \mathrm{H}^{\mathrm{E} 1}$ ), $7.69\left(\mathrm{dd}, J=8.5,1.6 \mathrm{~Hz}, 4 \mathrm{H}, \mathrm{H}^{\mathrm{E} 3}\right), 7.60\left(\mathrm{~d}, J=1.3 \mathrm{~Hz}, 4 \mathrm{H}, \mathrm{H}^{\mathrm{A} 5}\right)$, $7.58\left(\mathrm{~d}, J=8.2 \mathrm{~Hz}, 4 \mathrm{H}, \mathrm{H}^{\mathrm{E} 5 / \mathrm{E} 8}\right), 7.45\left(\mathrm{~d}, J=8.5 \mathrm{~Hz}, 4 \mathrm{H}, \mathrm{H}^{\mathrm{E} 4}\right), 7.43$ $\left(\mathrm{d}, J=1.3 \mathrm{~Hz}, 4 \mathrm{H}, \mathrm{H}^{\mathrm{A} 3}\right), 7.36\left(\mathrm{~d}, J=8.7 \mathrm{~Hz}, 8 \mathrm{H}, \mathrm{H}^{\mathrm{B} 2}\right), 7.33(\mathrm{~m}$, $\left.4 \mathrm{H}, \mathrm{H}^{\mathrm{E} 6 / \mathrm{E} 7}\right), 7.21\left(\mathrm{~m}, 8 \mathrm{H}, \mathrm{H}^{\mathrm{E} 5 / \mathrm{E} 8+\mathrm{E} 6 / \mathrm{E} 7}\right), 7.11\left(\mathrm{~m}, 56 \mathrm{H}, \mathrm{H}^{\mathrm{B} 3+\mathrm{C} 2+\mathrm{D} 2}\right)$, $6.95\left(\mathrm{~d}, J=8.9 \mathrm{~Hz}, 16 \mathrm{H}, \mathrm{H}^{\mathrm{C} 3}\right), 6.87\left(\mathrm{~d}, J=9.0 \mathrm{~Hz}, 32 \mathrm{H}, \mathrm{H}^{\mathrm{D} 3}\right), 3.79$ (s, 48H, H $\left.{ }^{\mathrm{OMe}}\right) .{ }^{13} \mathrm{C}$ NMR (126 MHz, $\left.\mathrm{CD}_{2} \mathrm{Cl}_{2}\right)$ d/ppm: $156.8\left(\mathrm{C}^{\mathrm{A} 6}\right)$, 156.6 $\left(\mathrm{C}^{\mathrm{D} 4}\right), 154.1\left(\mathrm{C}^{\mathrm{A} 2}\right), 151.0\left(\mathrm{C}^{\mathrm{B} 4}\right), 150.1\left(\mathrm{C}^{\mathrm{A} 4}\right), 146.2\left(\mathrm{C}^{\mathrm{C} 4}\right), 141.5$ $\left(\mathrm{C}^{\mathrm{D} 1}\right), 139.9\left(\mathrm{C}^{\mathrm{C} 1}\right), 136.4\left(\mathrm{C}^{\mathrm{E} 4 \mathrm{a} / \mathrm{E} 8 \mathrm{a}}\right), 133.7\left(\mathrm{C}^{\mathrm{E} 2}\right), 132.6\left(\mathrm{C}^{\mathrm{E} 4 \mathrm{a} / \mathrm{E} 8 \mathrm{a}}\right)$, $128.5\left(\mathrm{C}^{\mathrm{E} 5 / \mathrm{E} 8 / \mathrm{E} 6 / \mathrm{E} 7}\right), 128.2\left(\mathrm{C}^{\mathrm{B} 2}\right), 128.0\left(\mathrm{C}^{\mathrm{E} 1}\right), 127.8\left(\mathrm{C}^{\mathrm{E} 5 / \mathrm{E} 8}\right), 127.7$ $\left(\mathrm{C}^{\mathrm{E} 4+\mathrm{E} 6 / \mathrm{E} 7}\right), 127.5\left(\mathrm{C}^{\mathrm{B} 1}\right), 127.2\left(\mathrm{C}^{\mathrm{C} 2}\right), 127.0\left(\mathrm{C}^{\mathrm{D} 2+\mathrm{E} 6 / \mathrm{E} 7 / \mathrm{E} 5 / \mathrm{E} 8}\right), 125.6$ $\left(\mathrm{C}^{\mathrm{E} 3}\right), 122.1\left(\mathrm{C}^{\mathrm{C} 3}\right), 121.5\left(\mathrm{C}^{\mathrm{A} 5}\right) 120.0\left(\mathrm{C}^{\mathrm{B} 3}\right), 118.0\left(\mathrm{C}^{\mathrm{A} 3}\right), 115.2\left(\mathrm{C}^{\mathrm{D} 3}\right)$, $56.0\left(\mathrm{C}^{\mathrm{OMe}}\right)$. IR ( $\left(\tilde{v} / \mathrm{cm}^{-1}\right): 3036(\mathrm{w}), 3002(\mathrm{w}), 2991(\mathrm{w}), 2950(\mathrm{w})$, 2926 (w), 2903 (w), 2833 (w), 1592 (m), 1496 (s), 1314 (m), 1275 (m), $1261(\mathrm{~m}), 1237(\mathrm{~s}), 1033(\mathrm{~m}), 823$ (s), $765(\mathrm{~s}), 751$ (s), $575(\mathrm{~m})$, $525(\mathrm{~m}), 477(\mathrm{~m})$. MALDI-TOF MS (m/z): $3676.2\left[\mathrm{M}-\mathrm{PF}_{6}\right]^{+}$(calc. 3671.4), $1869.9[\mathrm{Cu}(\mathbf{1 2})]^{+}$(calc. 1867.7), $1805.9[12+\mathrm{H}]^{+}$(calc. 1804.8). UV-VIS $\left(\mathrm{CH}_{2} \mathrm{Cl}_{2}, 1.0 \times 10^{-5} \mathrm{~mol} \mathrm{dm}{ }^{-3}\right): \lambda_{\max } / \mathrm{nm}: 226$ ( $\left.\varepsilon / \mathrm{dm}^{3} \mathrm{~mol}^{-1} \mathrm{~cm}^{-1} 276400\right), 240 \mathrm{sh}$ (237 300), 306 (194 300), 340 sh (170 200), 448 (56 000), 580 sh (10 700). Found: C, 75.83; H, 5.42; N, 5.81; $\mathrm{C}_{244} \mathrm{H}_{196} \mathrm{~N}_{16} \mathrm{O}_{16} \mathrm{CuPF}_{6} \cdot 2 \mathrm{H}_{2} \mathrm{O}$ requires $\mathrm{C}$, 76.06; $\mathrm{H}$, $5.23 ; \mathrm{N}, 5.82(\%)$.

\section{Crystallography}

Single crystal data were collected on a Bruker APEX-II diffractometer with data reduction, solution and refinement using the programs APEX ${ }^{12}$ and SHELX-13. ${ }^{13}$ The ORTEP-type diagram and structure analysis used Mercury v. 3.0. ${ }^{\mathbf{1 4 , 1 5}}$

\section{Compound 3}

$\mathrm{C}_{58} \mathrm{H}_{58} \mathrm{~N}_{4} \mathrm{O}_{4}, M=875.08$, yellow block, triclinic, space group $P \overline{1}$, $a=9.9980(4), b=11.2625(4), c=11.6848(5) \AA, \alpha=64.9850(10)$, $\beta=80.227(2), \gamma=89.948(2)^{\circ}, U=1171.31(8) \AA^{3}, Z=1, D_{\mathrm{c}}=$ $1.241 \mathrm{Mg} \mathrm{m}^{-3}, \mu(\mathrm{Cu}-\mathrm{K} \alpha)=0.611 \mathrm{~mm}^{-1}, T=123 \mathrm{~K}$. Total 23653 reflections, 4153 unique, $R_{\text {int }}=0.0258$. Refinement of 3800 reflections (302 parameters) with $I>2 \sigma(I)$ converged at final $R 1=0.0343(R 1$ all data $=0.0376), \mathrm{w} R 2=0.0910(\mathrm{w} R 2$ all data $=$ 0.0942), gof $=1.040$. CCDC 987649 .

\section{DSC fabrication and measurements}

DSCs were prepared adapting the method of Grätzel and coworkers. ${ }^{\mathbf{1 6 , 1 7}}$ Solaronix Test Cell Titania Electrodes made from TCO22-7 FTO coated glass, prepared by screen-printing for a homogenous surface using Ti-Nanoxide pastes, active layer from Ti-Nanoxide $\mathrm{T} / \mathrm{SP}$ covered by a reflective layer of Ti-Nanoxide R/SP, active area: $6 \times 6 \mathrm{~mm}$, thickness: titania layer $9 \mu \mathrm{m}$ plus scattering layer $3 \mu \mathrm{m}$ (Fig. S1 $\dagger$ ) were used. The electrodes were rinsed with $\mathrm{EtOH}$ and sintered at $450{ }^{\circ} \mathrm{C}$ for $30 \mathrm{~min}$, then cooled to $c a .80{ }^{\circ} \mathrm{C}$ and immersed in a $1 \mathrm{mM}$ DMSO solution of the anchoring ligand 13 for $24 \mathrm{~h}$. The colourless electrode was removed from the solution, washed with DMSO and $\mathrm{EtOH}$ and dried at $60^{\circ} \mathrm{C}$. The functionalized electrode was immersed in either a $0.1 \mathrm{mM} \mathrm{CH}_{2} \mathrm{Cl}_{2}$ or a $0.1 \mathrm{mM}$ acetone solution of each homoleptic copper(I) complex for $\approx 68 \mathrm{~h}$. Reference cells were prepared by dipping a commercial electrode into an EtOH solution $(0.3 \mathrm{mM})$ of N719 (Solaronix) for $\approx 68 \mathrm{~h}$. The electrodes were finally washed with the same solvent as used for dye-assembly and dried at $60{ }^{\circ} \mathrm{C}$. Solaronix Test Cell Platinum Electrodes were used for the counterelectrodes, and residual organic impurities were removed by heating at $450{ }^{\circ} \mathrm{C}$ for $30 \mathrm{~min}$.

The dye-covered $\mathrm{TiO}_{2}$ electrode and $\mathrm{Pt}$ counter-electrode were assembled using thermoplast hot-melt sealing foil (Solaronix Test Cell Gaskets) by heating while pressing them together. The electrolyte (LiI $\left(0.1 \mathrm{~mol} \mathrm{dm}^{-3}\right), \mathrm{I}_{2}\left(0.05 \mathrm{~mol} \mathrm{dm}^{-3}\right)$, 1methylbenzimidazole $\left(0.5 \mathrm{~mol} \quad \mathrm{dm}^{-3}\right)$ and 1-butyl-3methylimidazolinium iodide $\left(0.6 \mathrm{~mol} \mathrm{dm}^{-3}\right)$ in 3-methoxypropionitrile) was introduced into the DSC by vacuum backfilling. The hole in the counter electrode was sealed using hotmelt sealing foil (Solaronix Test Cell Sealings) and a cover glass (Solaronix Test Cell Caps). Measurements were made by 
irradiating from behind using a light source SolarSim 150 (100 $\left.\mathrm{mW} \mathrm{cm}^{-2}=1 \mathrm{sun}\right)$. The power of the simulated light was calibrated by using a reference Si cell.

\section{Scanning electron microscopy}

Secondary electron SEM micrograph images were recorded under vacuum $\left(\sim 1 \times 10^{-6} \mathrm{mbar}\right)$ using an FEI Nova Nano SEM 230 at an accelerating voltage of $5 \mathrm{keV}$ and magnification of $\sim 9000 \times$. The sample was prepared by scoring and fracturing the glass electrode in order to image the cross section of the different layers.

\section{Results and discussion}

\section{Ligand synthesis and characterization}

We have previously described the syntheses of the first- and second-generation ligands 1 and 7 by treatment of $4,4^{\prime}$-bis(4bromophenyl)-6,6'-dimethyl-2,2'-bipyridine with either bis(4methoxyphenyl)amine or $4,4^{\prime}$-bis( $N, N$-bis(4-methoxyphenyl)amino)diphenylamine. ${ }^{8}$ An analogous strategy was adopted for the preparation of first-generation ligands 2-6 and secondgeneration ligands 8-12. The appropriate 4,4'-bis(4-bromophenyl)-6,6'-dialkyl-2,2'-bipyridine or 4,4'-bis(4-bromophenyl)$6,6^{\prime}$-diaryl-2,2'-bipyridine precursors have previously been reported $^{\mathbf{1 0}}$ with the exception of $4,4^{\prime}$-bis(4-bromophenyl)-6,6'di(naphthalen-2-yl)-2,2'-bipyridine (6a, Scheme 4). This was prepared by Krönhke methodology ${ }^{\mathbf{1 8}}$ starting from $(1 E, 5 E)-1,6$ bis(4-bromophenyl)hexa-1,5-diene-3,4-dione as shown in Scheme 4 . The product is poorly soluble in most common organic solvents and NMR spectra $†$ were recorded in TFA- $\mathrm{d}_{1}$ and thus represent data for the protonated ligand $[\mathrm{H}(\mathbf{6 a})]^{+}$. The ${ }^{1} \mathrm{H}$ and ${ }^{13} \mathrm{C}$ NMR spectra were assigned using COSY, NOESY, HMQC and HMBC methods. The resonance for $\mathrm{H}^{\mathrm{A} 3}$ is a sensitive probe for the conformation of the bpy domain. Neutral bpy

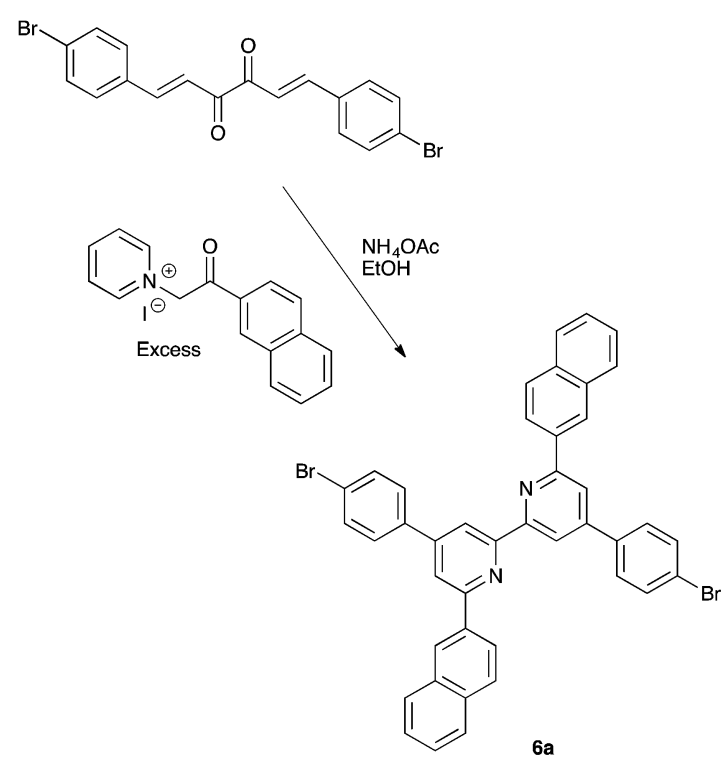

Scheme 4 Synthesis of the precursor 4,4'-bis(4-bromophenyl)-6,6'di(naphthalen-2-yl)-2,2' -bipyridine, $6 \mathrm{a}$, by Krönhke methodology. adopts a trans-configuration which switches to a cis-conformation in the monoprotonated ligand, and the resonance for proton $\mathrm{H}^{\mathrm{A} 5}$ (see Scheme 2 and $\mathrm{ESI} \dagger$ for atom numbering) is particularly sensitive to the protonation state of the bpy unit. ${ }^{19} \mathrm{~A}$ NOESY cross peak from $\mathrm{H}^{\mathrm{C} 1}$ to $\mathrm{H}^{\mathrm{A} 5}$ distinguishes the signals for $\mathrm{H}^{\mathrm{A} 5}$ ( $\left.\partial 8.66 \mathrm{ppm}\right)$ and $\mathrm{H}^{\mathrm{A} 3}(\partial 8.68 \mathrm{ppm})$. The close chemical shifts of these signals contrasts with their significant separation in 4,4'-bis(4-bromophenyl)-6, $6^{\prime}$-dialkyl-2,2'-bipyridines $\left(\mathrm{H}^{\mathrm{A} 3}\right.$ and $\mathrm{H}^{\mathrm{A} 5}$ appear at 28.48 and $7.34 \pm 0.03 \mathrm{ppm}$, respectively for alkyl $=\mathrm{Me},{ }^{n} \mathrm{Bu}$, ${ }^{\text {iso }} \mathrm{Bu},{ }^{n}$ hexyl) and in 4,4'-bis(4-bromophenyl)-6,6'diphenyl-2,2'-bipyridine ( $\partial 8.91$ and 8.04 ppm). ${ }^{\mathbf{8}, 10}$

The reactions shown in Scheme 2 proceeded smoothly to give, after purification, the first-generation ligands $\mathbf{2 - 5}$ in $>85 \%$ and 6 in $60 \%$. Yields for the second-generation compounds 8-12 ranged from 65 to $81 \%$. All the ligands are readily soluble in $\mathrm{CHCl}_{3}$ and $\mathrm{CH}_{2} \mathrm{Cl}_{2}$. The electrospray mass spectrum of each of 2-6 exhibited a base peak assigned to $[\mathrm{M}+\mathrm{H}]^{+}$. For the second-generation ligands, peaks were observed for $[\mathrm{M}+2 \mathrm{H}]^{2+}$ and, for 9 and 12, an additional peak arising from $[\mathrm{M}+\mathrm{H}]^{+} \cdot \dagger^{\dagger}$ The ${ }^{1} \mathrm{H}$ and ${ }^{13} \mathrm{C}$ NMR spectra of $\mathrm{CDCl}_{3}$ solutions of the compounds were assigned by $2 \mathrm{D}$ techniques. The spectra $\dagger$ indicate a symmetrical bpy domain and signal integrals are consistent with the bis-functionalized structures shown in Scheme 2. Representative spectra for a pair of first- and secondgeneration ligands are shown in Fig. 1.

$\mathrm{X}$-Ray quality single crystals of 3 were grown by $\mathrm{Et}_{2} \mathrm{O}$ diffusion into an acetone/chloroform solution of the compound. The structure of 3 is shown in Fig. 2. Selected bond parameters are given in the figure caption. The compound crystallizes in the space group $P \overline{1}$ with half of the molecule in the asymmetric unit; the second half is generated through an inversion centre and the bpy unit necessarily adopts a trans-conformation and is planar. The phenylene unit is twisted $36.7^{\circ}$ with respect to the pyridine ring to which it is bonded, thereby minimizing interring $\mathrm{H} \cdots \mathrm{H}$ interactions. Atom N2 is in a planar environment, consistent with delocalization of the lone pair into the arene $\pi$-systems. The twisted arrangement of the three arene rings bonded to N2 is expected on steric grounds. The presence of the

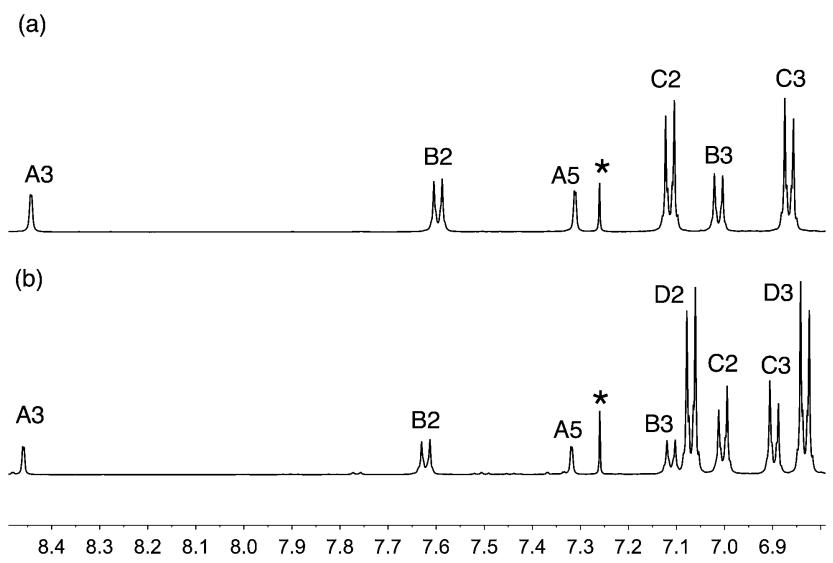

Fig. 1 Aromatic region of the $500 \mathrm{MHz}^{1} \mathrm{H}$ NMR spectra $\left(\mathrm{CDCl}_{3}, 295 \mathrm{~K}\right)$ of (a) first-generation 4 and (b) second-generation 10. See Scheme 2 and $\mathrm{ESI} \uparrow$ for ring labelling. ${ }^{*}=$ residual $\mathrm{CHCl}_{3}$. 


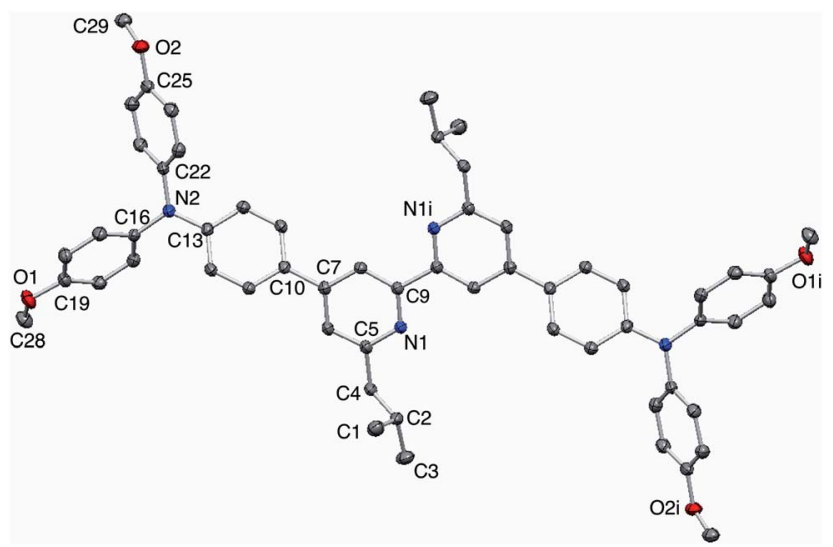

Fig. 2 Structure of compound 3 with ellipsoids plotted at $50 \%$ probability; $\mathrm{H}$ atoms omitted for clarity. Symmetry code $i=1-x, 2-y$, $2-z$. Selected bond parameters: N1-C5 =1.3451(14), N1-C9= $1.3453(14), \mathrm{N} 2-\mathrm{C} 13=1.4006(14), \mathrm{N} 2-\mathrm{C} 22=1.4262(14), \mathrm{N} 2-\mathrm{C} 16=$ $1.4302(14), \mathrm{C} 19-\mathrm{O} 1=1.3649(14), \mathrm{C} 25-\mathrm{O} 2=1.3697(14), \mathrm{O} 1-\mathrm{C} 28=$ $1.4225(16), \mathrm{O} 2-\mathrm{C} 29=1.4274(15) \AA ;$ C13-N2-C22 = 121.22(9), C13$\mathrm{N} 2-\mathrm{C} 16=120.38(9), \mathrm{C} 22-\mathrm{N} 2-\mathrm{C} 16=118.16(9), \mathrm{C} 19-\mathrm{O} 1-\mathrm{C} 28=$ $117.40(10), \mathrm{C} 25-\mathrm{O} 2-\mathrm{C} 29=116.05(9)^{\circ}$.

isobutyl groups prevents face-to-face interactions between bpy domains of neighbouring molecules. Dominant packing interactions involve methoxy $\mathrm{CH} \cdots \pi_{\text {pyridine }}$ contacts $(\mathrm{CH} \cdots$ centroid $=2.39 \AA$ ) which lead to a centrosymmetric embrace between adjacent molecules (Fig. 3a). These interactions lead to the assembly of hydrogen-bonded chains which slice obliquely through the unit cell (Fig. 3b).

The solution absorption spectra of the first- and secondgeneration ligands are displayed in Fig. 4 and 5, respectively. The spectra are all broad with intense bands which tail into the visible region. The observed absorptions originate from $\pi^{*} \leftarrow \pi$ and $\pi^{*} \leftarrow \mathrm{n}$ transitions, and the spectra of 1-4 (which contain 6- and $6^{\prime}$-alkyl substituents) are similar, as are those of the

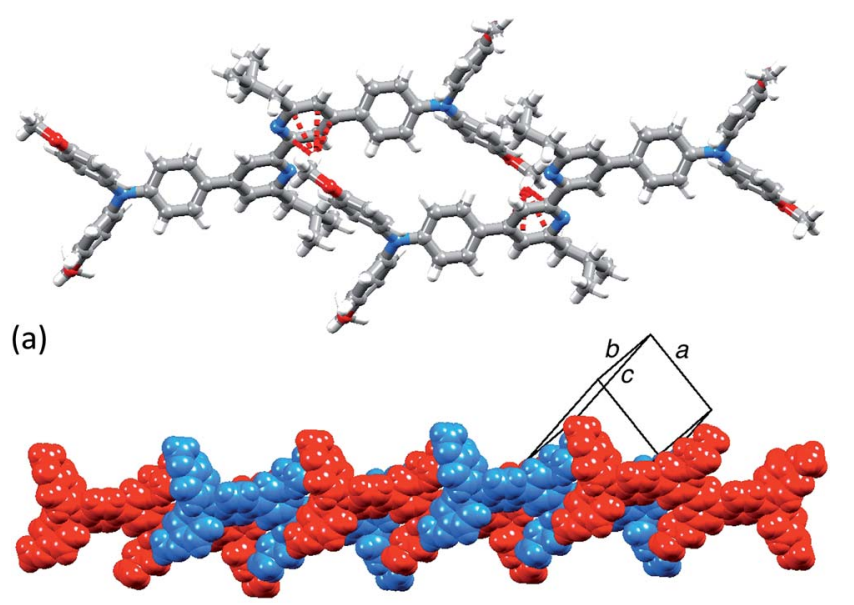

(b)

Fig. 3 (a) Centrosymmetric pair of molecules of 3 embrace through $\mathrm{CH}_{\mathrm{OMe}} \cdots \pi_{\text {pyridine }}$ contacts. (b) The interactions extend between molecules to generate hydrogen bonded chains.

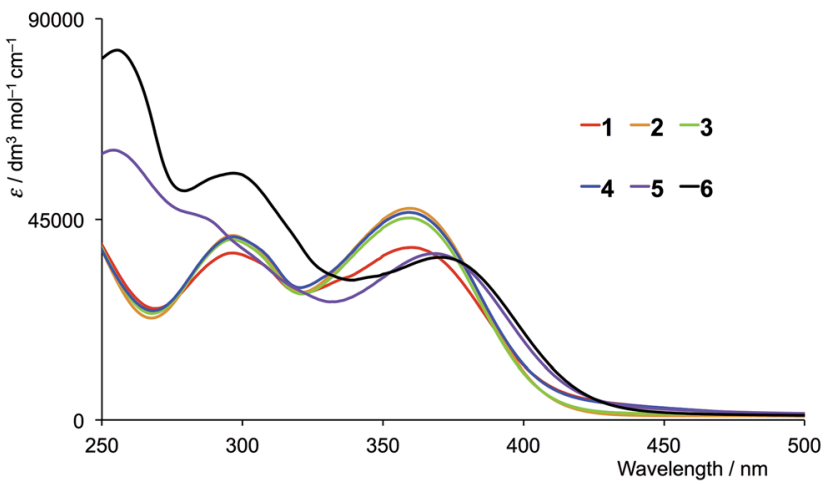

Fig. 4 Solution absorption spectra of the first-generation ligands 1-6 in $\mathrm{CH}_{2} \mathrm{Cl}_{2}\left(1 \times 10^{-5} \mathrm{~mol} \mathrm{dm}^{-3}\right)$.

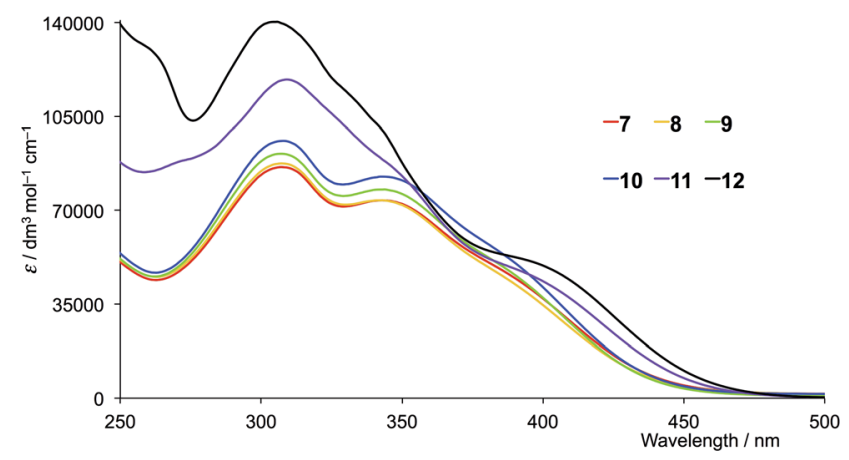

Fig. 5 Solution absorption spectra of the second-generation ligands 7-12 in $\mathrm{CH}_{2} \mathrm{Cl}_{2}\left(1 \times 10^{-5} \mathrm{~mol} \mathrm{dm}{ }^{-3}\right)$.

second-generation ligands 7-10. Extension of the aromatic domains on going from 1 to 7 (both with methyl substituents) leads to the anticipated increase in extinction coefficient (red curves in Fig. 4 and 5). Similar trends are observed upon comparing the spectra of 2 and 8,3 and $\mathbf{9}$, or 4 and $\mathbf{1 0}$. As expected, the intensities of the highest energy bands in the spectra of $\mathbf{5}$ and $\mathbf{1 1}$ (phenyl substituents, purple curves in Fig. 4 and 5) and 6 and 12 (naphthyl substituents, black curves in Fig. 4 and 5) are substantially larger than those of the alkylsubstituted ligands.

\section{Synthesis and characterization of homoleptic copper(I) complexes}

We have previously reported the syntheses and properties of $\left[\mathrm{Cu}(\mathbf{1})_{2}\right]\left[\mathrm{PF}_{6}\right]$ and $\left[\mathrm{Cu}(7)_{2}\right]\left[\mathrm{PF}_{6}\right]^{8}$ Homoleptic copper(I) complexes containing ligands 2-6 and 8-12 were prepared by treatment of $\left[\mathrm{Cu}(\mathrm{NCMe})_{4}\right]\left[\mathrm{PF}_{6}\right]$ with two equivalents of the appropriate ligand. Yields of $\left[\mathrm{CuL}_{2}\right]\left[\mathrm{PF}_{6}\right]$ were typically high. The electrospray mass spectra of the complexes containing the first-generation ligands 2-6 each exhibited a peak envelope corresponding to $\left[\mathrm{M}-\mathrm{PF}_{6}\right]^{+}$with the correct isotopic pattern. In addition, peaks assigned to $[3+\mathrm{H}]^{+},[4+\mathrm{H}]^{+},[5+\mathrm{H}]^{+}$or $[6+\mathrm{H}]^{+}$ were observed in the spectra of the respective complexes. Ligand loss was observed in the mass spectra of the complexes with the second-generation ligands and the spectra exhibited 
peaks for $[\mathrm{CuL}]^{+}$and $[\mathrm{L}+\mathrm{H}]^{+}$for $\mathrm{L}=\mathbf{8}, \mathbf{9 , 1 0}, 11$ or 12 . For $\mathrm{L}=\mathbf{9}$, 11 and 12, a peak envelope arising from $\left[\mathrm{M}-\mathrm{PF}_{6}\right]^{+}$was also observed.

The ${ }^{1} \mathrm{H}$ and ${ }^{13} \mathrm{C}$ NMR spectra of $\left[\mathrm{CuL}_{2}\right]\left[\mathrm{PF}_{6}\right]$ with $\mathrm{L}=\mathbf{2 - 6}$ were recorded in $\mathrm{CDCl}_{3}$, making the spectra directly comparable with those of the free ligands. Although the complexes containing the second-generation ligands are soluble in $\mathrm{CDCl}_{3}$, signals in the ${ }^{1} \mathrm{H}$ NMR spectra were broadened, necessitating a solvent change to $\mathrm{CD}_{2} \mathrm{Cl}_{2}$ to obtain well-resolved spectra. Fig. 6 compares the aromatic regions of the ${ }^{1} \mathrm{H}$ NMR spectra of 2 and $\left[\mathrm{Cu}(2)_{2}\right]\left[\mathrm{PF}_{6}\right]$. Shifts in the signals for $\mathrm{H}^{\mathrm{A} 3}$ and $\mathrm{H}^{\mathrm{A} 5}$ are consistent with the change in conformation of the bpy unit upon coordination. Resonances for the peripheral dendron protons are essentially unaffected; the signal for the OMe protons appears at $\delta 3.82 \mathrm{ppm}$ in 2 and at $\delta 3.83 \mathrm{ppm}$ in $\left[\mathrm{Cu}(2)_{2}\right]\left[\mathrm{PF}_{6}\right]$. The trends are typical of the spectra of all ligand-complex pairs in which the ligand bears 6- and 6'-alkyl substituents (Table 1). The shift to lower frequency for $\mathrm{H}^{\mathrm{A} 3}$ on comparing ligand with complex (Table 1) is more pronounced for ligands 5 and 11 (phenyl substituents) than for the pairs of alkyl-substituted ligands, and is even more so for $\mathbf{6}$ and $\mathbf{1 2}$ (2-naphthyl substituents). The structure of $\left[\mathrm{Cu}(6)_{2}\right]^{+}$modelled using Spartan 14 (v. 1.1.3, MMFF level) is shown in Fig. 7. The $\mathrm{H}^{\mathrm{A} 3}$ protons on one bpy ligand are located within a cleft between the two naphthyl domains of the second ligand and thus lie in the shielding region of their ring currents. The same effect is observed for both first- and secondgeneration ligands and their complexes (Table 1).

The absorption spectra of $\mathrm{CH}_{2} \mathrm{Cl}_{2}$ solutions of the copper(I) complexes are shown in Fig. 8 (first-generation ligands) and Fig. 9 (second-generation). The broad absorption bands extend significantly further to longer wavelength than for the free ligands. The approximate doubling of the extinction coefficients on comparing the spectra of ligands with complexes is consistent with the homoleptic complexes $\left[\mathrm{CuL}_{2}\right]^{+}$. At high energies $(\lambda<450 \mathrm{~nm})$, the absorption spectra of $\left[\mathrm{CuL}_{2}\right]^{+}$for $\mathrm{L}=1-6$ possess similar band-shapes to those of the first-generation ligands 1-6 (compare Fig. 8 with Fig. 4). The ligand-centred band at $\approx 360 \mathrm{~nm}$ undergoes a red-shift of $10-30 \mathrm{~nm}$ on

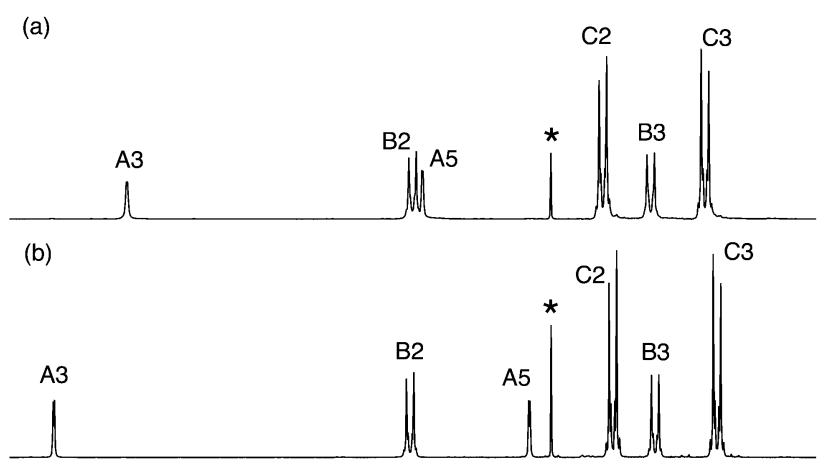

$\begin{array}{lllllllllllllllllllllll}8.5 & 8.4 & 8.3 & 8.2 & 8.1 & 8.0 & 7.9 & 7.8 & 7.7 & 7.6 & 7.5 & 7.4 & 7.3 & 7.2 & 7.1 & 7.0 & 6.9 & 6.8 & 6.7\end{array}$

Fig. 6 Aromatic region of the $500 \mathrm{MHz}^{1} \mathrm{H}$ NMR spectra $\left(\mathrm{CDCl}_{3}, 295 \mathrm{~K}\right)$ of (a) $\left[\mathrm{Cu}(2)_{2}\right]\left[\mathrm{PF}_{6}\right]$ and (b) free ligand 2 . See Scheme 2 and $\mathrm{ESI}$ † for ring labelling. ${ }^{*}=$ residual $\mathrm{CHCl}_{3}$.
Table 1 Comparison of chemical shifts of bpy protons $\mathrm{H}^{\mathrm{A} 3}$ and $\mathrm{H}^{\mathrm{A} 5}$ in free ligand, $\mathrm{L}$, in $\mathrm{CDCl}_{3}$ and $\left[\mathrm{CuL}_{2}\right]\left[\mathrm{PF}_{6}\right]$ complexes in $\mathrm{CDCl}_{3}$ for $\mathrm{L}=1-6$ and $C D_{2} \mathrm{Cl}_{2}$ for $\mathrm{L}=7-12$

\begin{tabular}{lccll}
\hline & & & & \\
$\mathrm{L}$ & $\mathrm{H}^{\mathrm{A} 3}$ in $\mathrm{L}$ & $\mathrm{H}^{\mathrm{A} 5}$ in $\mathrm{L}$ & $\begin{array}{l}\mathrm{H}^{\mathrm{A} 3} \text { in } \\
{\left[\mathrm{CuL}_{2}\right]^{+}}\end{array}$ & $\begin{array}{l}\mathrm{H}^{\mathrm{A} 5} \text { in } \\
{\left[\mathrm{CuL}_{2}\right]^{+}}\end{array}$ \\
\hline \multicolumn{2}{l}{ First-generation } & & & \\
$\mathbf{1}$ & 8.39 & 7.33 & 8.25 & 7.59 \\
$\mathbf{2}$ & 8.44 & 7.31 & 8.27 & 7.57 \\
$\mathbf{3}$ & 8.46 & 7.28 & 8.27 & 7.52 \\
$\mathbf{4}$ & 8.44 & 7.31 & 8.26 & 7.56 \\
$\mathbf{5}$ & 8.82 & 7.95 & 7.95 & 7.61 \\
$\mathbf{6}$ & 8.89 & 8.11 & 7.32 & 7.57 \\
& & & & \\
$\mathbf{5}$ & & & & \\
$\mathbf{7}$ & 8.40 & 7.33 & 8.26 & 7.57 \\
$\mathbf{8}$ & 8.46 & 7.32 & 8.34 & 7.60 \\
$\mathbf{9}$ & 8.46 & 7.28 & 8.35 & 7.56 \\
$\mathbf{1 0}$ & 8.46 & 7.32 & 8.34 & 7.61 \\
$\mathbf{1 1}$ & 8.83 & 7.96 & 8.02 & 7.66 \\
$\mathbf{1 2}$ & 8.93 & 8.12 & 7.43 & 7.60 \\
& & & &
\end{tabular}

going from ligand to complex. The shoulder at around $480 \mathrm{~nm}$ in the spectra of $\left[\mathrm{CuL}_{2}\right]^{+}$for $\mathrm{L}=\mathbf{1 - 4}$ is absent in the spectra of the ligands and is assigned to the MLCT band. The energy is consistent with the band at $483 \mathrm{~nm}$ observed in $\left[\mathrm{Cu}\left(6,6-\mathrm{Me}_{2} \mathrm{bpy}\right)_{2}\right]^{+}$, the MLCT character of which has been confirmed from TD-DFT calculations. ${ }^{20}$ For $\left[\mathrm{Cu}(5)_{2}\right]\left[\mathrm{PF}_{6}\right]$ and $\left[\mathrm{Cu}(6)_{2}\right]\left[\mathrm{PF}_{6}\right]$, a low intensity band at 560 and $576 \mathrm{~nm}$, respectively, (Fig. 8) is assumed to arise from the MLCT. The absorption spectra of $\left[\mathrm{CuL}_{2}\right]^{+}$for second-generation ligands 7-12 are dominated by high energy bands originating from ligand-based $\pi^{*} \leftarrow \pi$ and $\pi^{*} \leftarrow \mathrm{n}$ transitions (Fig. 9). Broad shoulders in the spectra of $\mathrm{CH}_{2} \mathrm{Cl}_{2}$ solutions of $\left[\mathrm{Cu}(7)_{2}\right]\left[\mathrm{PF}_{6}\right],\left[\mathrm{Cu}(8)_{2}\right]\left[\mathrm{PF}_{6}\right]$, $\left[\mathrm{Cu}(\mathbf{9})_{2}\right]\left[\mathrm{PF}_{6}\right]$ and $\left[\mathrm{Cu}(\mathbf{1 0})_{2}\right]\left[\mathrm{PF}_{6}\right]$ centred around $480 \mathrm{~nm}$ are assigned to MLCT transitions. (Note that the previously reported spectrum for $\left[\mathrm{Cu}(7)_{2}\right]\left[\mathrm{PF}_{6}\right]$ with $\lambda_{\max }=505 \mathrm{~nm}$ was recorded in MeCN. $)^{8}$ The MLCT bands for $\left[\mathrm{Cu}(\mathbf{1 1})_{2}\right]\left[\mathrm{PF}_{6}\right]$ and $\left[\mathrm{Cu}\left(\mathbf{1 2}_{2}\right]\left[\mathrm{PF}_{6}\right]\right.$ are assigned to the low intensity absorption maxima at $580 \mathrm{~nm}$ (Fig. 9), consistent with the first-generation analogues (Fig. 8). The broad and intense spectral responses of

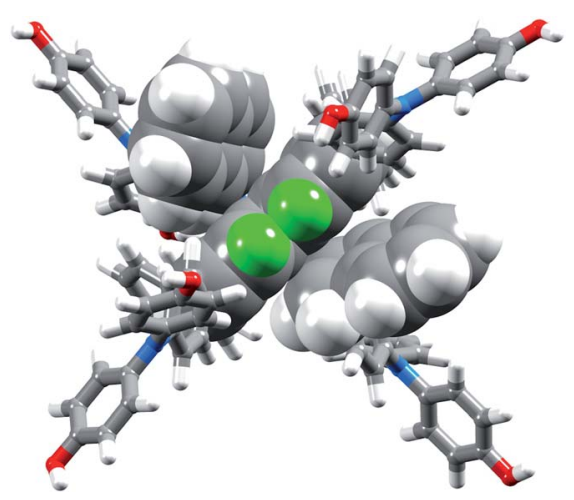

Fig. 7 Modelled structure of $\left[\mathrm{Cu}(6)_{2}\right]^{+}$. The $\mathrm{H}^{\mathrm{A} 3}$ protons (in green) and bpy domain of one ligand and the two 2-naphthyl groups of the second ligand are shown in space-filling representation. 


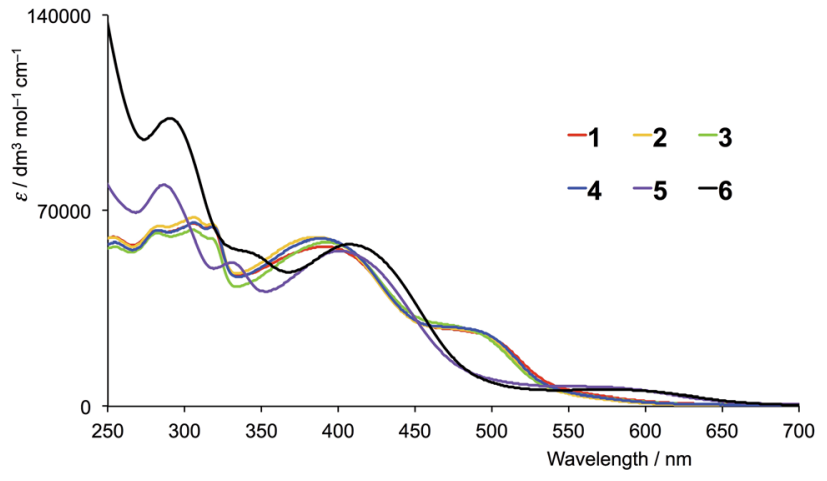

Fig. 8 Solution absorption spectra of in $\mathrm{CH}_{2} \mathrm{Cl}_{2}$ solutions of $\left(1 \times 10^{-5}\right.$ mol dm${ }^{-3}$ ) $\left[\mathrm{CuL}_{2}\right]\left[\mathrm{PF}_{6}\right]$ where $\mathrm{L}=1-6$ (first-generation ligands).

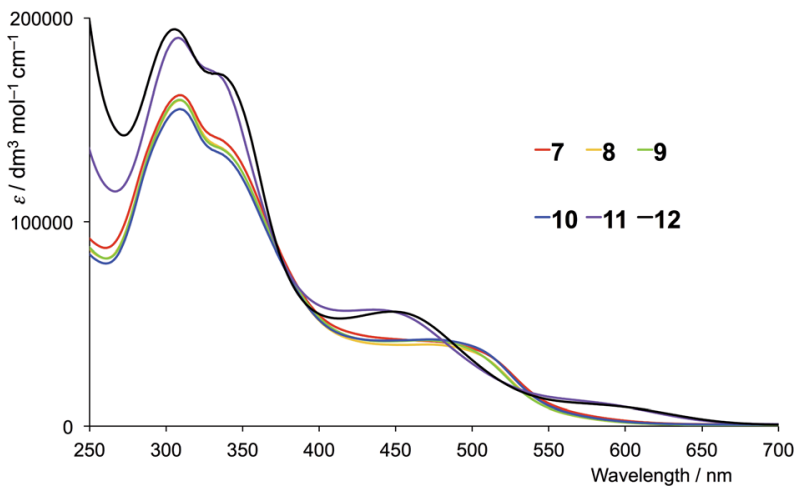

Fig. 9 Solution absorption spectra of in $\mathrm{CH}_{2} \mathrm{Cl}_{2}$ solutions of $\left(1 \times 10^{-5}\right.$ $\mathrm{mol} \mathrm{dm}{ }^{-3}$ ) $\left[\mathrm{CuL}_{2}\right]\left[\mathrm{PF}_{6}\right]$ where $\mathrm{L}=7-12$ (second-generation ligands).

all the complexes, especially those containing ligands 7-12, suggest that they should be good candidates as precursors to dyes in DSCs.

\section{Electrochemistry}

Cyclic voltammetric data for the homoleptic copper(I) complexes are presented in Table 2. Cyclic voltammograms were recorded $\mathrm{CH}_{2} \mathrm{Cl}_{2}$ to preclude association of the metal ion with coordinating solvents, ${ }^{21}$ and are always referenced to internal ferrocene. For the first- or second-generation complexes, three or five oxidation processes are observed, respectively. A representative pair of CVs for first-generation $\left.[\mathrm{Cu}(3)]_{2}\right]\left[\mathrm{PF}_{6}\right]$ and second-generation $\left[\mathrm{Cu}(\mathbf{9})_{2}\right]\left[\mathrm{PF}_{6}\right]$ is shown in Fig. 10. By comparison with related systems, the oxidation process in the range $0.40-0.58 \mathrm{~V}$ is assigned to the $\mathrm{Cu}^{+} / \mathrm{Cu}^{2+}$ couple. In copper(I) diimine complexes, incorporation of substituents ortho to the $\mathrm{N}$-donor sites (e.g. 6,6'-positions in bpy) stabilizes tetrahedral $\mathrm{Cu}^{+}$with respect to square-planar copper(II) by sterically encumbering the flattening of the coordination environment. The potentials for the $\mathrm{Cu}^{+} / \mathrm{Cu}^{2+}$ couple in $\left[\mathrm{CuL}_{2}\right]\left[\mathrm{PF}_{6}\right]$ with $\mathrm{L}=\mathbf{1 - 6}$ follow the same trend as in the series $\left[\mathrm{CuL}_{2}\right]\left[\mathrm{PF}_{6}\right]$ with $\mathrm{L}=\mathbf{7 - 1 2}$. The complexes containing the phenyl or 2-naphthyl substituents (ligands 5, 6, 11 and 12) exhibit the lowest metal oxidation potentials, followed by the complexes containing the methyl substituents (ligands 1 and 7). The metalcentred oxidation in $\left[\mathrm{Cu}(2)_{2}\right]\left[\mathrm{PF}_{6}\right],\left[\mathrm{Cu}(3)_{2}\right]\left[\mathrm{PF}_{6}\right],\left[\mathrm{Cu}(4)_{2}\right]\left[\mathrm{PF}_{6}\right]$, $\left[\mathrm{Cu}(\mathbf{8})_{2}\right]\left[\mathrm{PF}_{6}\right],\left[\mathrm{Cu}(\mathbf{9})_{2}\right]\left[\mathrm{PF}_{6}\right]$ and $\left[\mathrm{Cu}(\mathbf{1 0})_{2}\right]\left[\mathrm{PF}_{6}\right]$ (n-butyl, isobutyl or $n$-hexyl substituents) occurs at similar potentials $(+0.56 \pm$ $0.02 \mathrm{~V}$ ), noticeably higher than in the methyl, phenyl or 2naphthyl-containing complexes. The trends are consistent with data for $\left[\mathrm{CuL}_{2}\right]\left[\mathrm{PF}_{6}\right]$ with $\mathrm{L}=4,4^{\prime}$-bis(4-bromophenyl)-6,6'-dialkyl-2,2'-bipyridines (alkyl $=\mathrm{Me},{ }^{n} \mathrm{Bu},{ }^{\text {iso }} \mathrm{Bu},{ }^{n}$ hexyl) or bis(4bromophenyl)-6,6'-diphenyl-2,2'-bipyridine. ${ }^{10}$ For the alkyl substituents, the trend follows the steric bulk of the group, with the complexes containing the least sterically demanding substituents being the easiest to oxidize. We have previously commented ${ }^{10}$ that the metal centre in $\left[\mathrm{CuL}_{2}\right]^{+}$with $\mathrm{L}=4,4^{\prime}$ bis(4-bromophenyl)-6, 6'-diphenyl-2,2'-bipyridine possesses a flattened geometry in the solid state as a consequence of $\pi$-stacking of 6-phenyl groups of one ligand and the bpy domain of the other. This leads to entatic ${ }^{22}$ lower energy metal oxidation as the coordination geometry is already enroute to that favoured by $\mathrm{Cu}^{2+}$.

For complexes containing the first-generation ligands, a ligand-based oxidation process is observed close to $+0.30 \mathrm{~V}$ similar to that observed in the free ligand. ${ }^{8}$ For $\left[\mathrm{CuL}_{2}\right]\left[\mathrm{PF}_{6}\right]$ complexes with second-generation ligands $\mathbf{7 - 1 2}$, four ligandcentred oxidation processes are observed in each complex (Table 2), again assigned by analogy with the free ligand. ${ }^{8}$ The potential for each ligand oxidation process shows little variation across the series $\left[\mathrm{Cu}(7)_{2}\right]\left[\mathrm{PF}_{6}\right]$ to $\left[\mathrm{Cu}(\mathbf{1 2})_{2}\right]\left[\mathrm{PF}_{6}\right]$.

Ligand reduction processes in the complexes lie close to the edge of the solvent accessible window and are typically poorly defined. Each complex exhibits an irreversible reduction process close to $-2.05 \mathrm{~V}$ which appears only in the first cycle (Fig. 10).

\section{DSC fabrication and solid-state absorption spectra}

Heteroleptic dyes for DSC measurements were assembled by stepwise adsorption of anchoring ligand 13 onto commercial titania electrodes followed by treatment with a solution of the labile ${ }^{23,24}$ homoleptic complexes $\left[\mathrm{CuL}_{2}\right]\left[\mathrm{PF}_{6}\right](\mathrm{L}=\mathbf{1}$ to 12$)$. Ligand exchange occurs with the formation of surface-bound heteroleptic dyes (Scheme 5). Our strategy for surfaceimmobilized dye assembly ${ }^{\mathbf{8 - 1 0 , 2 0}}$ has recently been employed by Robertson and coworkers. ${ }^{25}$ In the present study, we observed that dye uptake is dependent on the solvent used during the dipping process. Fig. 11 shows the appearance of DSCs which have been assembled using $\mathrm{CH}_{2} \mathrm{Cl}_{2}$ or acetone solutions of $\left[\mathrm{CuL}_{2}\right]\left[\mathrm{PF}_{6}\right](\mathrm{L}=\mathbf{1}$ to 12$)$. In every pair of $\mathrm{CH}_{2} \mathrm{Cl}_{2}$ /acetone dipped cells, the intensity of colour was reproducibly greater for the acetone-dipping.

FTO/ $\mathrm{TiO}_{2}$ electrodes (without a scattering layer) with adsorbed dye were prepared and their solid-state absorption spectra recorded, each spectrum being corrected for the background spectrum of a blank electrode. Absorption spectra for electrodes with first-generation dyes made using $\mathrm{CH}_{2} \mathrm{Cl}_{2}$ or acetone solutions of the homoleptic complexes $\left[\mathrm{CuL}_{2}\right]\left[\mathrm{PF}_{6}\right]$ with $\mathrm{L}=\mathbf{1 - 6}$ are shown in Fig. 12a and b, respectively. For a given ancillary ligand, the general trend is for enhanced absorptivity when 
Table 2 Cyclic voltammetric data for $\left[\mathrm{CuL}_{2}\right]\left[\mathrm{PF}_{6}\right]$ with $\mathrm{L}=1-12$ with respect to $\mathrm{Fc} / \mathrm{Fc}^{+} ; \mathrm{CH}_{2} \mathrm{Cl}_{2}$ solutions with $\left[{ }^{n} \mathrm{Bu}_{4} \mathrm{~N}\right]\left[\mathrm{PF}_{6}\right]$ as supporting electrolyte and scan rate of $0.1 \mathrm{~V} \mathrm{~s}^{-1}$. Processes are reversible unless otherwise stated (qr = quasi-reversible; ir = irreversible)

\begin{tabular}{|c|c|c|c|c|c|c|}
\hline Complex & $\begin{array}{l}E_{1 / 2}{ }^{\mathrm{ox}} / \mathrm{V} \\
\left(E_{\mathrm{pc}}-E_{\mathrm{pa}} / \mathrm{mV}\right)\end{array}$ & $\begin{array}{l}E_{1 / 2}{ }^{\mathrm{ox}} / \mathrm{V} \\
\left(E_{\mathrm{pc}}-E_{\mathrm{pa}} / \mathrm{mV}\right)\end{array}$ & $\begin{array}{l}E_{1 / 2}{ }^{\mathrm{ox}} / \mathrm{V} \\
\left(E_{\mathrm{pc}}-E_{\mathrm{pa}} / \mathrm{mV}\right)\end{array}$ & $\begin{array}{l}E_{1 / 2}{ }^{\mathrm{ox}} / \mathrm{V} \\
\left(E_{\mathrm{pc}}-E_{\mathrm{pa}} / \mathrm{mV}\right)\end{array}$ & $E_{1 / 2}^{\mathrm{ox}} / \mathrm{V}\left(E_{\mathrm{pc}}-E_{\mathrm{pa}} / \mathrm{mV}\right)$ & $\begin{array}{l}E_{1 / 2}^{\mathrm{red}} / \mathrm{V} \\
\left(E_{\mathrm{pc}}-E_{\mathrm{pa}} / \mathrm{mV}\right)\end{array}$ \\
\hline$\left[\mathrm{Cu}(\mathbf{1})_{2}\right]\left[\mathrm{PF}_{6}\right]$ & & $+0.29(96)$ & $+0.44(87)$ & & $+1.00^{\mathrm{ir}}$ & $-2.04^{\mathrm{ir}}$ \\
\hline$\left[\mathrm{Cu}(3)_{2}\right]\left[\mathrm{PF}_{6}\right]$ & & $+0.29(86)$ & $+0.58(66)$ & & $+0.99(102)^{\mathrm{qr}}$ & $-2.07^{\mathrm{ir}}$ \\
\hline$\left[\mathrm{Cu}(4)_{2}\right]\left[\mathrm{PF}_{6}\right]$ & & $+0.29(89)$ & $+0.57(74)$ & & $+0.96(106)^{\mathrm{qr}}$ & $-2.08^{\mathrm{ir}}$ \\
\hline$\left[\mathrm{Cu}(5)_{2}\right]\left[\mathrm{PF}_{6}\right]$ & & $+0.32^{a}$ & $+0.40^{a}$ & & $+0.99(83)^{\mathrm{qr}^{\mathrm{r}}}$ & $-2.25^{\mathrm{ir}}$ \\
\hline$\left[\mathrm{Cu}(\mathbf{8})_{2}\right]\left[\mathrm{PF}_{6}\right]$ & $-0.07(83)$ & $+0.19(83)$ & $+0.54(99)$ & $+0.69(155)$ & $+1.01(111)^{\mathrm{qr}}$ & $-2.07^{\mathrm{ir}}$ \\
\hline$\left[\mathrm{Cu}(\mathbf{9})_{2}\right]\left[\mathrm{PF}_{6}\right]$ & $-0.08(65)$ & $+0.18(88)$ & $+0.55(90)$ & $+0.68(157)$ & $+1.02(85)^{\mathrm{qr}}$ & $-2.06^{\mathrm{ir}}$ \\
\hline$\left[\mathrm{Cu}(\mathbf{1 0})_{2}\right]\left[\mathrm{PF}_{6}\right]$ & $-0.07(79)$ & $+0.18(89)$ & $+0.55(84)$ & $+0.69(150)$ & $+1.03(86)^{\mathrm{qr}}$ & $-2.06^{\mathrm{ir}}$ \\
\hline$\left[\mathrm{Cu}(\mathbf{1 1})_{2}\right]\left[\mathrm{PF}_{6}\right]$ & $-0.07(81)$ & $+0.19(103)$ & $+0.39(81)$ & $+0.69(170)$ & $+1.03(69)^{\mathrm{qr}}$ & $-2.19^{\mathrm{ir}}$ \\
\hline$\left[\mathrm{Cu}(\mathbf{1 2})_{2}\right]\left[\mathrm{PF}_{6}\right]$ & $-0.08(63)$ & $+0.19(85)$ & $+0.39(67)$ & $+0.68(168)$ & $+1.02^{\text {ir }}$ & $-2.08^{\mathrm{ir}}$ \\
\hline
\end{tabular}

${ }^{a} 1$ st and 2nd oxidation processes overlap.

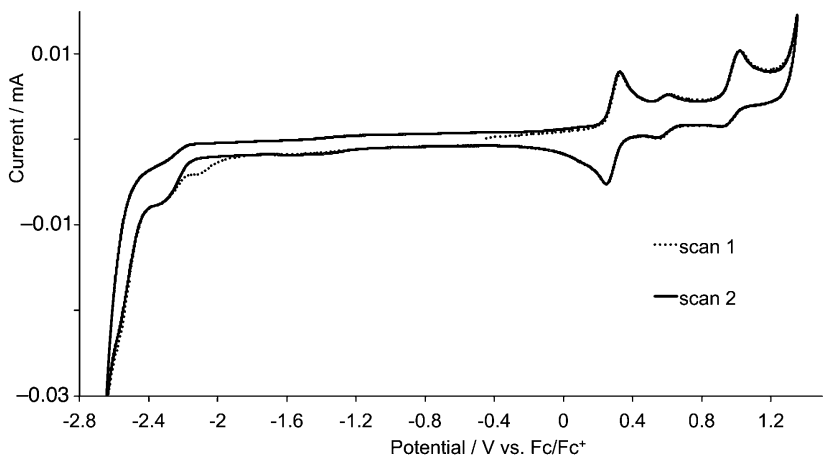

(a)

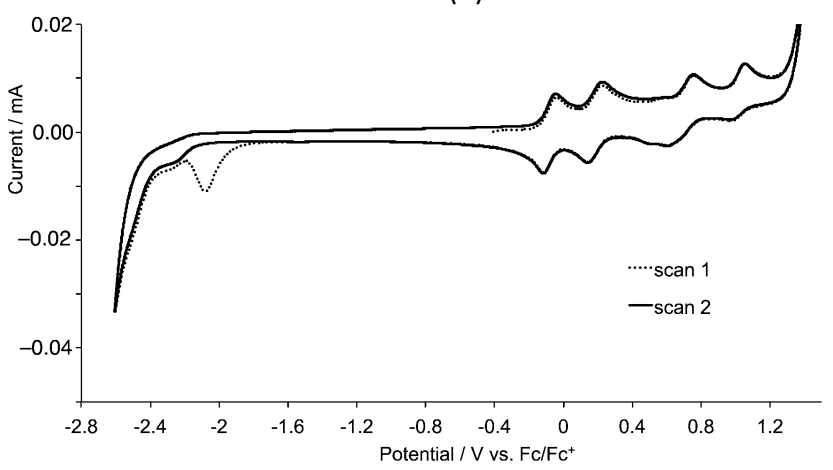

(b)

Fig. 10 Cyclic voltammograms of $\mathrm{CH}_{2} \mathrm{Cl}_{2}$ (degassed) solutions of (a) $\left[\mathrm{Cu}(3)_{2}\right]\left[\mathrm{PF}_{6}\right]$ and $(\mathrm{b})\left[\mathrm{Cu}(9)_{2}\right]\left[\mathrm{PF}_{6}\right]$ for first- and second cycles. Scan rate $=0.1 \mathrm{Vs}^{-1}$.

acetone is used in the dipping solution during cell fabrication. A similar trend is observed for complexes containing the secondgeneration ligands 7-12. The enhancement of absorption observed on changing from $\mathrm{CH}_{2} \mathrm{Cl}_{2}$ to acetone coupled with that on going from first- to second-generation ligand in the surfaceimmobilized heteroleptic complex, is exemplified in Fig. 13 with $[\mathrm{Cu}(\mathbf{1 3})(\mathbf{4})]^{+}$and $[\mathrm{Cu}(\mathbf{1 3})(\mathbf{1 0})]^{+}$.

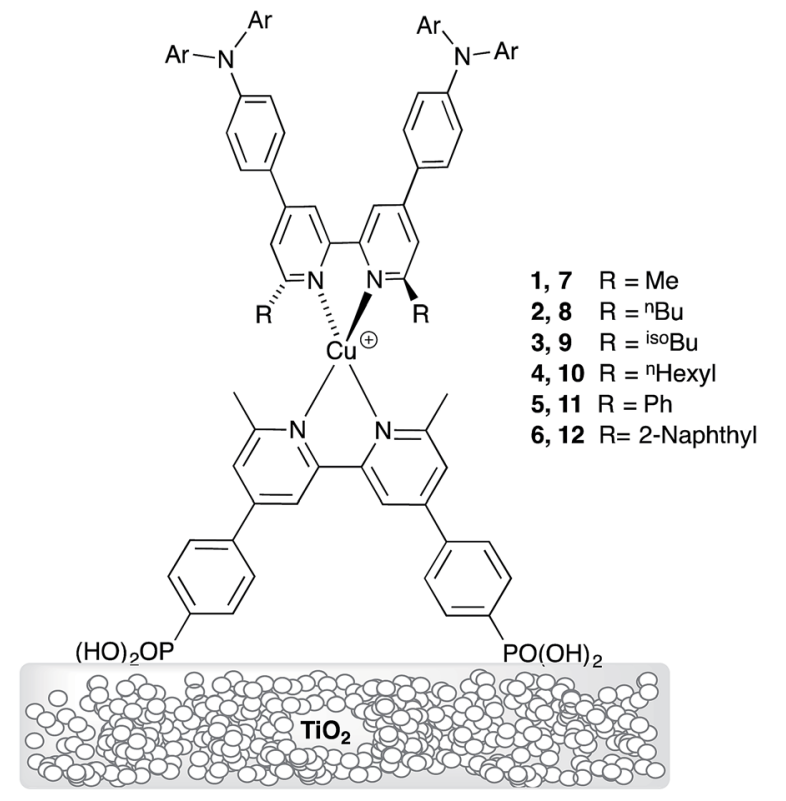

Scheme 5 Anchored heteroleptic dyes. For the first-generation, $\mathrm{Ar}=$ 4- $\mathrm{MeOC}_{6} \mathrm{H}_{4}$ in ligands 1-6 (see Scheme 2); for the second-generation, $\mathrm{Ar}=4-\mathrm{MeOC}_{6} \mathrm{H}_{4}$ in ligands 7-12 (see Scheme 2).

DSC performances: first- versus second-generation ancillary ligands

The enhanced absorption observed using acetone in the dye dipping cycle leads us to initially focus on the performances of these DSCs. All DSCs were masked to avoid overestimation of efficiencies. $^{26}$ In order to ensure that DSC parameters were reproducible, measurements were made using duplicate cells for each dye-solvent combination. A complete set of DSC parameters is presented in Table $\mathrm{S} 1, \uparrow$ and Table 3 summarizes representative DSC characteristics using anchoring ligand 13, first-generation ancillary ligands and acetone in the dye dipping cycle, measured over a period of three weeks and with respect to the standard dye N719. The final column in the table shows 


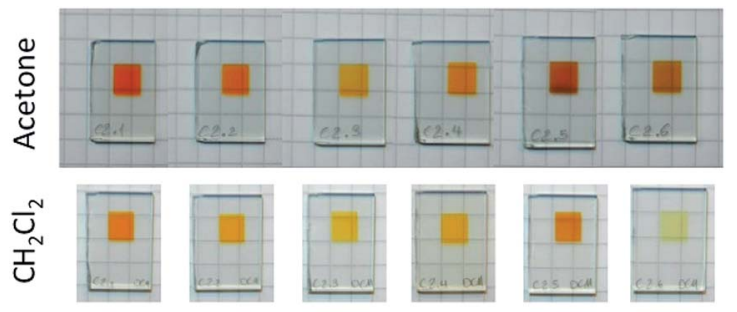

(a)

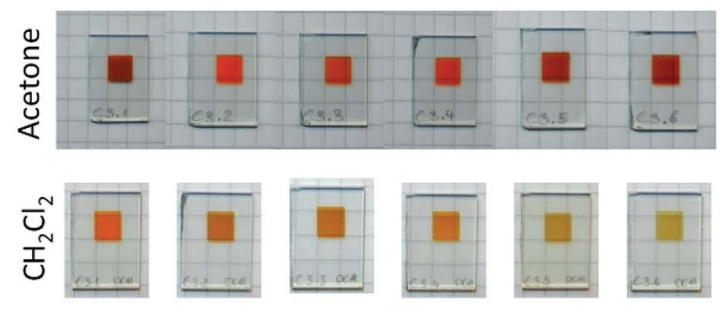

(b)

Fig. 11 Cells after dipping in acetone or $\mathrm{CH}_{2} \mathrm{Cl}_{2}$ solutions of the homoleptic complexes: (a) first-generation ligands 1-6 (left to right), (b) second-generation ligands 7-12 (left to right). (a)

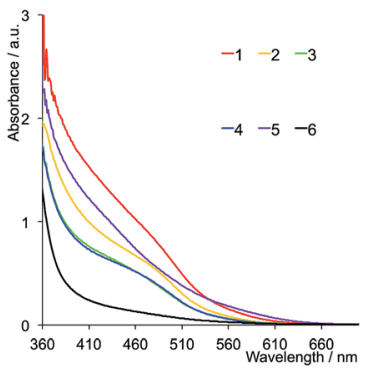

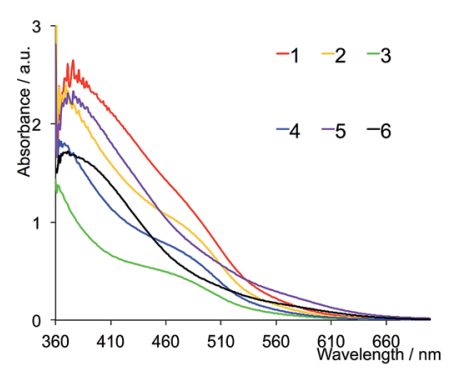

(b)
Fig. 12 Solid-state absorption spectra of electrodes with anchored dyes $[\mathrm{Cu}(13)(\mathrm{L})]^{+}$where $\mathrm{L}=1-6$. The electrodes were made using (a) $\mathrm{CH}_{2} \mathrm{Cl}_{2}$, or (b) acetone solutions of $\left[\mathrm{CuL}_{2}\right]\left[\mathrm{PF}_{6}\right]$.

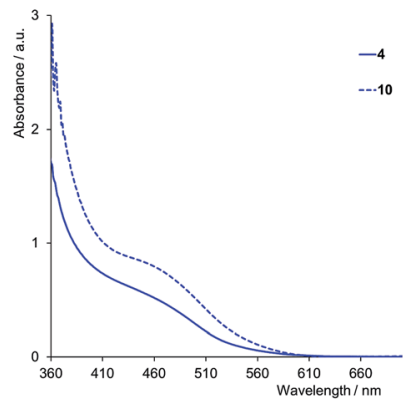

(a)

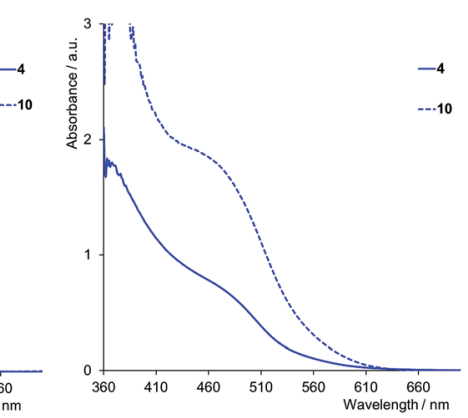

(b)
Fig. 13 Solid-state absorption spectra of electrodes with the anchored first- and second-generation dyes $[\mathrm{Cu}(13)(4)]^{+}$and $[\mathrm{Cu}(13)(10)]^{+}$. The electrodes with anchored dye were prepared using (a) $\mathrm{CH}_{2} \mathrm{Cl}_{2}$, or (b) acetone solutions of $\left[\mathrm{CuL}_{2}\right]\left[\mathrm{PF}_{6}\right](\mathrm{L}=4$ or 10$)$.

relative efficiencies with respect to N719 set to $100 \%$. Performance data for the dyes containing the second-generation ligands 7-12 are given in Tables 4 and S2.†
Table 3 DSC performance data for sealed and masked cells using anchoring ligand 13 and first-generation ancillary ligands, and acetone in the $\left[\mathrm{CuL}_{2}\right]\left[\mathrm{PF}_{6}\right]$ dipping cycle. Relative efficiencies (last column) are with respect to $100 \%$ for standard dye N719 measured under the same conditions. See also ESI Table S1†

\begin{tabular}{llllll}
\hline Anchored dye & $J_{\mathrm{SC}} / \mathrm{mA} \mathrm{cm}^{-2}$ & $V_{\mathrm{OC}} / \mathrm{mV}$ & ff & $\eta / \%$ & Relative $\eta / \%$ \\
\hline \multicolumn{2}{l}{ On the day of sealing the cell } \\
{$[\mathrm{Cu}(\mathbf{1 3})(\mathbf{1})]^{+}$} & 6.00 & 510 & 70.3 & 2.15 & 29.4 \\
{$[\mathrm{Cu}(\mathbf{1 3})(2)]^{+}$} & 4.41 & 482 & 69.4 & 1.48 & 20.2 \\
{$[\mathrm{Cu}(\mathbf{1 3})(\mathbf{3})]^{+}$} & 4.22 & 475 & 69.6 & 1.39 & 19.0 \\
{$[\mathrm{Cu}(\mathbf{1 3})(\mathbf{4})]^{+}$} & 5.60 & 542 & 71.8 & 2.18 & 29.8 \\
{$[\mathrm{Cu}(\mathbf{1 3})(\mathbf{5})]^{+}$} & 3.22 & 468 & 68.6 & 1.03 & 14.1 \\
{$[\mathrm{Cu}(\mathbf{1 3})(\mathbf{6})]^{+}$} & 4.29 & 508 & 67.0 & 1.46 & 19.9 \\
$\mathrm{~N} 719$ & 16.72 & 641 & 68.4 & 7.32 & 100
\end{tabular}

$\mathbf{2 2}^{a}$ days after sealing the cell

Anchored dye $\quad J_{\mathrm{SC}} / \mathrm{mA} \mathrm{cm}^{-2} \quad V_{\mathrm{OC}} / \mathrm{mV} \quad$ ff $\quad \eta / \% \quad$ Relative $\eta / \%$ $\begin{array}{llllll}{[\mathrm{Cu}(13)(1)]^{+}} & 5.27 & 520 & 71.1 & 1.94 & 24.4\end{array}$ $\begin{array}{llllll}{[\mathrm{Cu}(13)(2)]^{+}} & 4.42 & 516 & 70.7 & 1.61 & 20.3\end{array}$ $\begin{array}{llllll}{[\mathrm{Cu}(13)(3)]^{+}} & 4.18 & 487 & 69.6 & 1.42 & 17.9\end{array}$ $\begin{array}{llllll}{[\mathrm{Cu}(13)(4)]^{+}} & 5.05 & 532 & 70.8 & 1.90 & 23.9\end{array}$ $\begin{array}{llllll}{[\mathrm{Cu}(13)(5)]^{+}} & 4.00 & 485 & 69.9 & 1.36 & 17.1\end{array}$ $\begin{array}{llllll}{[\mathrm{Cu}(13)(6)]^{+}} & 4.10 & 504 & 68.0 & 1.41 & 17.7\end{array}$ $\begin{array}{llllll}\text { N719 } & 16.65 & 671 & 71.2 & 7.95 & 100\end{array}$

${ }^{a}$ For $[\mathrm{Cu}(\mathbf{1 3})(\mathbf{1})]^{+}$, the second measurements were made after 18 days.

A comparison of Tables 3 and 4 reveals that, with the exception of $[\mathrm{Cu}(\mathbf{1 3})(\mathbf{6})]^{+}$and $[\mathrm{Cu}(\mathbf{1 3})(\mathbf{1 2})]^{+}\left(\mathbf{6}\right.$ and $\mathbf{1 2}=6,6^{\prime}$-bis $(2-$ naphthyl) substituted ligands), the dyes containing the secondgeneration ancillary ligands give higher global efficiencies. This corresponds to an increase in the solid-state absorbance of the

Table 4 DSC performance data for masked cells using anchoring ligand 13 and second-generation ancillary ligands, and acetone in the $\left[\mathrm{CuL}_{2}\right]\left[\mathrm{PF}_{6}\right]$ dipping cycle. Relative efficiencies (last column) are with respect to $100 \%$ for standard dye N719 measured under the same conditions. See also ESI Table S2†

\begin{tabular}{lcllll}
\hline Anchored dye & $J_{\mathrm{SC}} / \mathrm{mA} \mathrm{cm}^{-2}$ & $V_{\mathrm{OC}} / \mathrm{mV}$ & ff & $\eta / \%$ & Relative $\eta / \%$ \\
\hline \multicolumn{7}{l}{ On the day of sealing the cell } \\
{$[\mathrm{Cu}(\mathbf{1 3})(7)]^{+}$} & 6.46 & 515 & 67.9 & 2.26 & 32.8 \\
{$[\mathrm{Cu}(\mathbf{1 3})(\mathbf{8})]^{+}$} & 6.08 & 506 & 70.8 & 2.18 & 31.6 \\
{$[\mathrm{Cu}(\mathbf{1 3})(\mathbf{9})]^{+}$} & 5.48 & 475 & 69.9 & 1.82 & 26.4 \\
{$[\mathrm{Cu}(\mathbf{1 3})(\mathbf{1 0})]^{+}$} & 5.25 & 488 & 70.9 & 1.81 & 26.2 \\
{$[\mathrm{Cu}(\mathbf{1 3})(\mathbf{1 1})]^{+}$} & 4.01 & 459 & 70.2 & 1.29 & 18.7 \\
{$[\mathrm{Cu}(\mathbf{1 3})(\mathbf{1 2})]^{+}$} & 3.04 & 444 & 69.2 & 0.93 & 13.5 \\
$\mathrm{~N} 719$ & 16.52 & 608 & 68.8 & 6.90 & 100
\end{tabular}

$\mathbf{2 2}^{a}$ days after sealing the cell Anchored dye $\quad J_{\mathrm{SC}} / \mathrm{mA} \mathrm{cm}^{-2} \quad V_{\mathrm{OC}} / \mathrm{mV} \quad$ ff $\quad \eta / \% \quad$ Relative $\eta / \%$ $\begin{array}{llllll}{[\mathrm{Cu}(13)(7)]^{+}} & 5.94 & 536 & 70.3 & 2.23 & 27.5\end{array}$ $\begin{array}{llllll}{[\mathrm{Cu}(13)(8)]^{+}} & 5.97 & 532 & 71.6 & 2.27 & 28.0\end{array}$ $\begin{array}{llllll}{[\mathrm{Cu}(13)(9)]^{+}} & 6.31 & 541 & 71.0 & 2.42 & 29.8\end{array}$ $\begin{array}{llllll}{[\mathrm{Cu}(13)(10)]^{+}} & 5.47 & 522 & 70.6 & 2.02 & 24.9\end{array}$ $\begin{array}{llllll}{[\mathrm{Cu}(13)(11)]^{+}} & 4.99 & 487 & 71.3 & 1.73 & 21.3\end{array}$ $\begin{array}{llllll}{[\mathrm{Cu}(13)(12)]^{+}} & 2.77 & 466 & 70.2 & 0.91 & 11.2\end{array}$ $\begin{array}{llllll}\text { N719 } & 16.98 & 674 & 70.9 & 8.11 & 100\end{array}$

${ }^{a}$ For N719, the second measurement was made after 15 days. 
DSCs as illustrated in Fig. 12. After three weeks, the DSCs with the ancillary ligands 7-9 and $\mathbf{1 1}$ exhibit higher $J_{\mathrm{SC}}$ values than those with 1-3 and 5 (Fig. 14, 15, S1 and S2 $\dagger$ ), while $J_{\mathrm{SC}}$ for dyes containing the $n$-hexyl subtituents (4 and 10) are similar (Fig. S3†). For $[\mathrm{Cu}(\mathbf{1 3})(\mathbf{9})]^{+}$(isobutyl substituents, secondgeneration), there is also a significant gain in $V_{\mathrm{OC}}$ over the three week period (Fig. 14). The dyes with the methyl (Fig. 15) or $n$-butyl (Fig. S1†) substituents perform the best, with enhancement on going from first- to second-generation ancillary ligands. $[\mathrm{Cu}(\mathbf{1 3})(\mathbf{1})]^{+}$and $[\mathrm{Cu}(\mathbf{1 3})(7)]^{+}$(methyl substituted ancillary ligands) suffer from reduced $J_{\mathrm{SC}}$ as the DSCs age, but benefit from increased $V_{\text {OC }}$. This latter enhancement is also observed in $[\mathrm{Cu}(\mathbf{1 3})(\mathbf{2})]^{+}$and $[\mathrm{Cu}(\mathbf{1 3})(\mathbf{8})]^{+}$(n-butyl groups).

The dyes which incorporate the sterically hindering 2naphthyl groups exhibit poorer device performances than the other dyes, and this is associated with lower $J_{\mathrm{SC}}$ values (Fig. 16). As the DSCs age, the efficiencies decrease, with this trend observed for both generations of ligands; these observations are confirmed for the duplicate DSCs (Tables S1 and S2 $\dagger$ ). The data also suggest that extending the dendron on going from 6 to 12 is detrimental to overall dye performance (Fig. 16).

Fig. 17 shows the EQE spectra of freshly sealed DSCs for all the dyes; the spectra were also recorded after 22 days (Fig. S4 and $55 \dagger$ ). Only small changes in EQE parameters (Tables 5 and 6 ) are observed for a given DSC over a 22 day period. Except for 2-naphthyl-containing dyes, on going from the first- to secondgeneration ligand in each pair $\left(\right.$ e.g. $[\mathrm{Cu}(\mathbf{1 3})(\mathbf{1})]^{+}$to $\left.[\mathrm{Cu}(\mathbf{1 3})(\mathbf{7})]^{+}\right)$, values of $\mathrm{EQE}_{\max }$ typically increase. Irrespective of the ancillary ligand, the dyes exhibit values of $\lambda_{\max }$ in the range $470-490 \mathrm{~nm}$. For the dye containing 1 (methyl groups), Fig. 17a shows a pronounced low energy shoulder at $590 \mathrm{~nm}$, confirming improved photoresponse and electron injection consistent with the relatively high conversion efficiency observed for anchored dye $[\mathrm{Cu}(\mathbf{1 3})(\mathbf{1})]^{+}$(Table 4). The dyes with the second-generation alkyl-functionalized ancillary ligands all exhibit enhanced EQE to lower energies than the first-generation analogues (Fig. 17b versus 17a). This observation appears not to carry through to dyes $\mathbf{5}$ and $\mathbf{1 1}$ with phenyl substituents where there is little gain in EQE at higher wavelength upon extending the hole transport

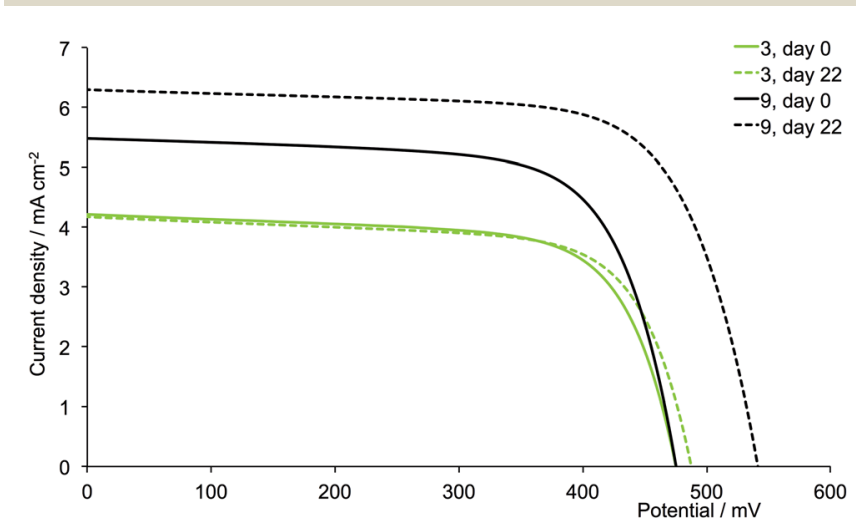

Fig. $14 \mathrm{~J}-V$ curves for dyes $[\mathrm{Cu}(13)(3)]^{+}$and $[\mathrm{Cu}(13)(9)]^{+}$(isobutyl substituents, acetone used in the dye-dipping cycle) on day of sealing the DSC (day 0 ) and after 18 or 22 days showing enhancement of $J_{\text {SC }}$ with second-generation ligand.

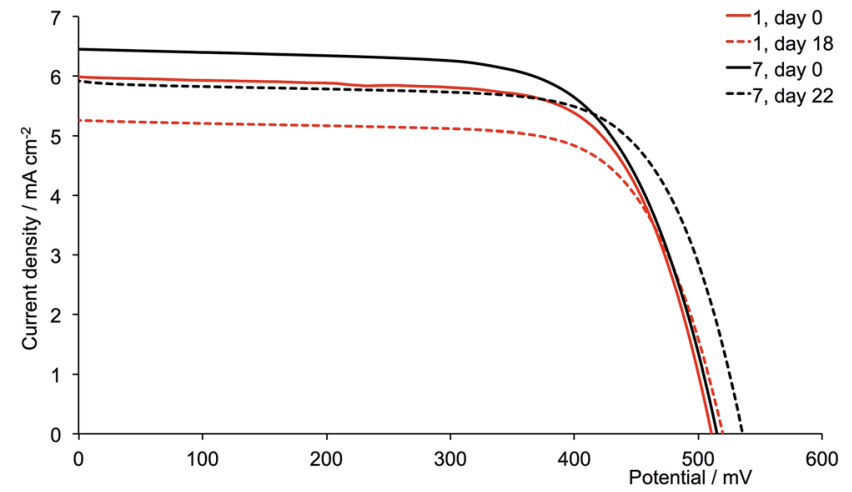

Fig. $15 \mathrm{~J}-V$ curves for dyes $[\mathrm{Cu}(13)(1)]^{+}$and $[\mathrm{Cu}(13)(7)]^{+}$(methyl substituents, acetone used in the $\left[\mathrm{CuL}_{2}\right]\left[\mathrm{PF}_{6}\right]$ dipping cycle) on day of sealing the DSC (day 0 ) and after 18 or 22 days.

domain. Consistent with the earlier discussion, EQE data confirm poorer electron injection on going from 6 to 12 (naphthyl-groups).

\section{DSC performances: influence of solvent in the dye dipping cycles}

We now return to the influence of the solvent during the dye dipping cycle when making the solar cells. Tables S3 and S4† give DSC parameters for cells (including duplicates) which were fabricated using $\mathrm{CH}_{2} \mathrm{Cl}_{2}$ solutions of the homoleptic dyes. Measurements were made over a three week period and Fig. S6 and $\mathrm{S} 7 \dagger$ summarize the $J-V$ characteristics. Data for the best performing cells on the day of sealing are presented in Table 7 , and Fig. 18 and 19 show $J-V$ curves for the first- and secondgeneration dyes, respectively. A comparison of the data in Table 7 with those in Tables 3 and 4 indicates that, in general, the conversion efficiencies are higher when acetone is used during cell fabrication. It is difficult to see unambiguous trends between the DSC performances and the 6,6'-substituents in the ancillary ligand. However, in all cases, the dyes containing the methyl substituents perform well. The worst performing dyes contain the bulky 2-naphthyl groups, irrespective of solvent,

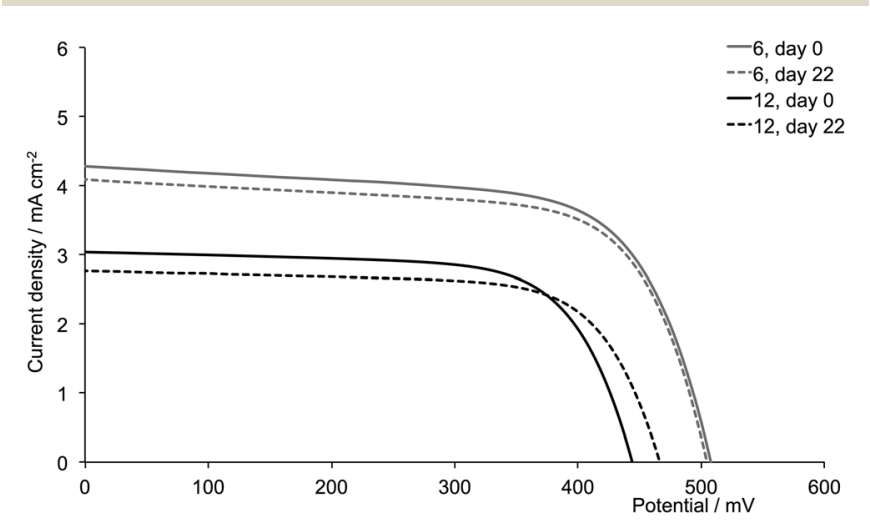

Fig. $16 \mathrm{~J}-\mathrm{V}$ curves for dyes $[\mathrm{Cu}(13)(6)]^{+}$and $[\mathrm{Cu}(13)(12)]^{+}$(naphthyl substituents, acetone used in the $\left[(\mathrm{CuL})_{2}\right]\left[\mathrm{PF}_{6}\right]$ dipping cycle) on day of sealing the DSC (day 0 ) and after 22 days. 


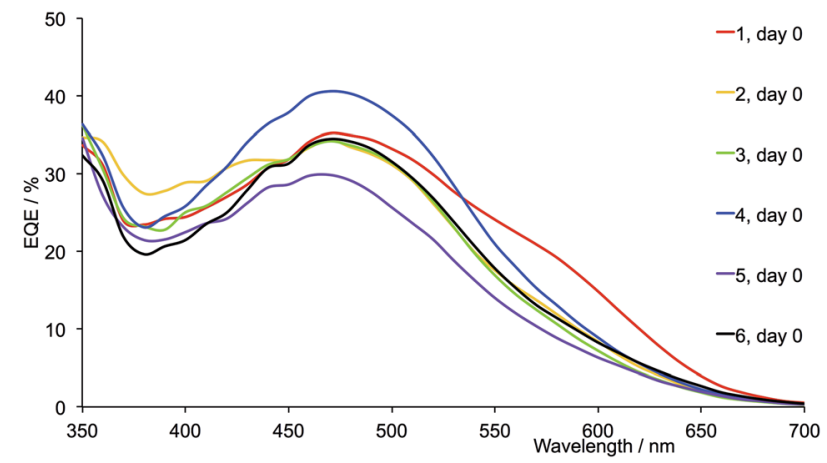

(a)

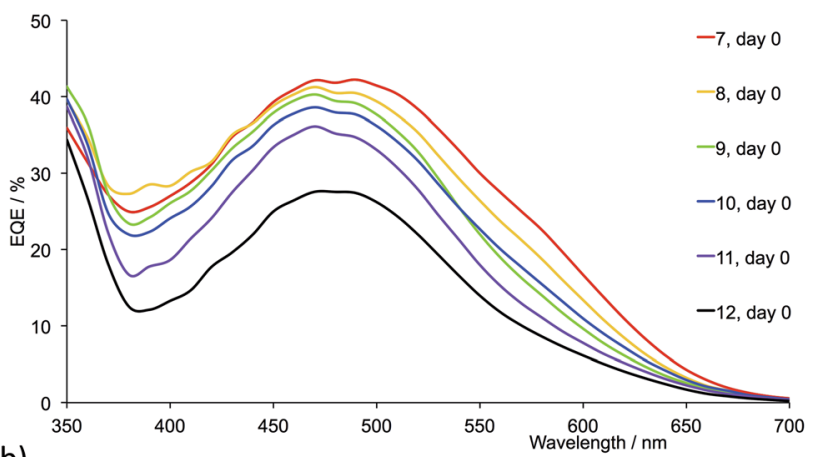

(b)

Fig. 17 EQE spectra (on the day of sealing) of DSCs with dyes containing ancillary ligands (a) 1-6 and (b) 7-12. Acetone was used in the $\left[\mathrm{CuL}_{2}\right]\left[\mathrm{PF}_{6}\right]$ dipping cycle.

Table 5 EQE maxima for DSCs containing first-generation dyes

\begin{tabular}{llllll}
\hline & Day 0 & & & Day 22 & \\
\cline { 2 - 3 } \cline { 5 - 6 } Anchored dye & $\lambda_{\max } / \mathrm{nm}$ & $\mathrm{EQE}_{\max } / \%$ & & $\lambda_{\text {max }} / \mathrm{nm}$ & $\mathrm{EQE}_{\text {max }} / \%$ \\
\hline$[\mathrm{Cu}(\mathbf{1 3})(\mathbf{1})]^{+}$ & 470 & 35.2 & 470 & 31.3 \\
{$[\mathrm{Cu}(\mathbf{1 3})(\mathbf{2})]^{+}$} & 470 & 34.4 & 470 & 32.8 \\
{$[\mathrm{Cu}(\mathbf{1 3})(\mathbf{3})]^{+}$} & 470 & 34.2 & & 470 & 32.0 \\
{$[\mathrm{Cu}(\mathbf{1 3})(\mathbf{4})]^{+}$} & 470 & 40.6 & & 470 & 37.2 \\
{$[\mathrm{Cu}(\mathbf{1 3})(\mathbf{5})]^{+}$} & 470 & 29.0 & 470 & 32.7 \\
{$[\mathrm{Cu}(\mathbf{1 3})(\mathbf{6})]^{+}$} & 470 & 35.0 & 470 & 31.2 \\
\hline
\end{tabular}

Table 6 EQE maxima for DSCs containing second-generation dyes

\begin{tabular}{llllll}
\hline & Day 0 & & & Day 22 & \\
\cline { 2 - 3 } \cline { 5 - 6 } Anchored dye & $\lambda_{\text {max }} / \mathrm{nm}$ & $\mathrm{EQE}_{\text {max }} / \%$ & & $\lambda_{\text {max }} / \mathrm{nm}$ & $\mathrm{EQE}_{\text {max }} / \%$ \\
\hline$[\mathrm{Cu}(\mathbf{1 3})(7)]^{+}$ & $470-490$ & 42.2 & & 480 & 41.8 \\
{$[\mathrm{Cu}(\mathbf{1 3})(\mathbf{8})]^{+}$} & 470 & 41.3 & & 470 & 41.7 \\
{$[\mathrm{Cu}(\mathbf{1 3})(\mathbf{9})]^{+}$} & 470 & 40.3 & & 470 & 46.5 \\
{$[\mathrm{Cu}(\mathbf{1 3})(\mathbf{1 0})]^{+}$} & 470 & 38.6 & & 480 & 41.3 \\
{$[\mathrm{Cu}(\mathbf{1 3})(\mathbf{1 1})]^{+}$} & 470 & 36.1 & & 480 & 41.4 \\
{$[\mathrm{Cu}(\mathbf{1 3})(\mathbf{1 2})]^{+}$} & $460-470$ & 27.6 & 470 & 24.3 \\
\hline
\end{tabular}

and the EQE spectra in Fig. 17 and 20 confirm the poorest electron injection as a function of wavelength for dyes with ligands 6 and 12.
Table 7 DSC performance data for masked cells using anchoring ligand 13 and first or second-generation ancillary ligands, and $\mathrm{CH}_{2} \mathrm{Cl}_{2}$ in the $\left[\mathrm{CuL}_{2}\right]\left[\mathrm{PF}_{6}\right]$ dipping cycle. Relative efficiencies (last column) are with respect to $100 \%$ for standard dye N719 measured under the same conditions. See also ESI Tables S3 and S4 $\uparrow$

\begin{tabular}{llllll}
\hline Anchored dye & $J_{\mathrm{SC}} / \mathrm{mA} \mathrm{cm}^{-2}$ & $V_{\mathrm{OC}} / \mathrm{mV}$ & ff & $\eta / \%$ & Relative $\eta / \%$ \\
\hline
\end{tabular}

First-generation ligands; on the day of sealing the cell

$\begin{array}{llllll}{[\mathrm{Cu}(13)(\mathbf{1})]^{+}} & 5.47 & 490 & 69.4 & 1.86 & 25.1 \\ {[\mathrm{Cu}(\mathbf{1 3})(\mathbf{2})]^{+}} & 4.65 & 475 & 69.8 & 1.54 & 20.8 \\ {[\mathrm{Cu}(\mathbf{1 3})(\mathbf{3})]^{+}} & 3.94 & 479 & 69.6 & 1.31 & 17.7 \\ {[\mathrm{Cu}(\mathbf{1 3})(\mathbf{4})]^{+}} & 4.07 & 491 & 71.8 & 1.43 & 19.3 \\ {[\mathrm{Cu}(\mathbf{1 3})(\mathbf{5})]^{+}} & 5.35 & 492 & 70.4 & 1.85 & 25.0 \\ {[\mathrm{Cu}(13)(\mathbf{6})]^{+}} & 2.94 & 485 & 68.3 & 0.97 & 13.1 \\ \mathrm{~N} 719 & 16.31 & 637 & 71.3 & 7.41 & 100.0\end{array}$

Second-generation ligands; on the day of sealing the cell Anchored dye $\quad J_{\mathrm{SC}} / \mathrm{mA} \mathrm{cm}^{-2} \quad V_{\mathrm{OC}} / \mathrm{mV} \quad$ ff $\quad \eta / \% \quad$ Relative $\eta / \%$ $\begin{array}{llllll}{[\mathrm{Cu}(13)(7)]^{+}} & 4.32 & 509 & 68.1 & 1.50 & 20.3\end{array}$ $\begin{array}{llllll}{[\mathrm{Cu}(13)(8)]^{+}} & 4.08 & 469 & 67.9 & 1.30 & 17.6\end{array}$ $\begin{array}{llllll}{[\mathrm{Cu}(13)(9)]^{+}} & 2.60 & 428 & 65.3 & 0.73 & 9.9\end{array}$ $\begin{array}{llllll}{[\mathrm{Cu}(13)(10)]^{+}} & 2.73 & 459 & 68.5 & 0.86 & 11.6\end{array}$ $\begin{array}{llllll}{[\mathrm{Cu}(13)(11)]^{+}} & 1.78 & 418 & 67.2 & 0.50 & 6.8\end{array}$ $\begin{array}{llllll}{[\mathrm{Cu}(13)(12)]^{+}} & 1.63 & 411 & 61.1 & 0.41 & 5.5\end{array}$ $\begin{array}{llllll}\mathrm{N} 719 & 16.44 & 647 & 69.5 & 7.40 & 100.0\end{array}$

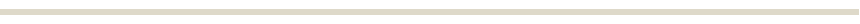

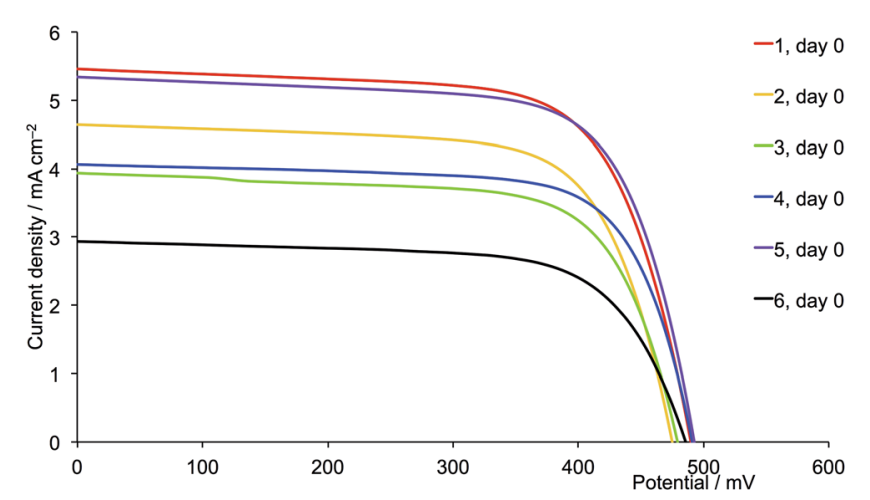

Fig. $18 J-V$ curves for dyes $[\mathrm{Cu}(13)(\mathrm{L})]^{+}$with $\mathrm{L}=1-6$ on day of sealing the DSCs (day 0). $\mathrm{CH}_{2} \mathrm{Cl}_{2}$ was used in the $\left[\mathrm{CuL}_{2}\right]\left[\mathrm{PF}_{6}\right]$ dipping cycle.

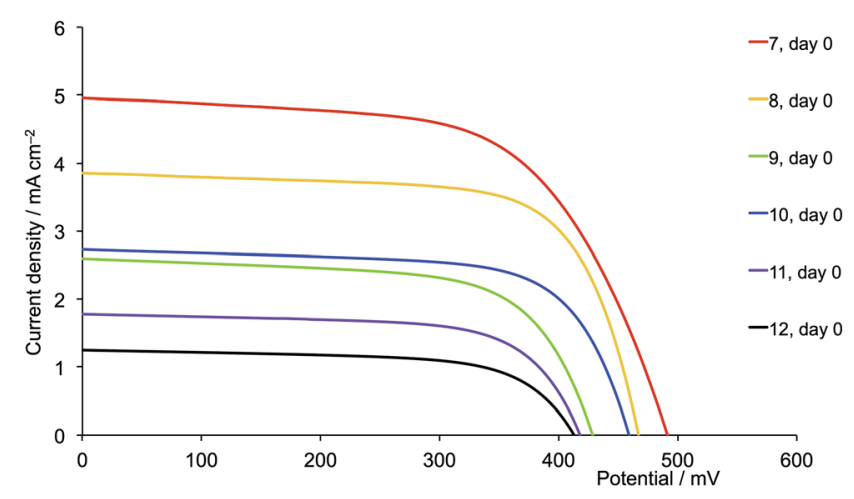

Fig. $19 \mathrm{~J}-\mathrm{V}$ curves for dyes $[\mathrm{Cu}(13)(\mathrm{L})]^{+}$with $\mathrm{L}=7-12$ on day of sealing the DSCs (day 0 ). $\mathrm{CH}_{2} \mathrm{Cl}_{2}$ was used in the $\left[\mathrm{CuL}_{2}\right]\left[\mathrm{PF}_{6}\right]$ dipping cycle. 
For the second-generation dyes (Fig. 19), the DSC performance corresponds quite well to the steric demands of the $6,6^{\prime}$ substituents, the best $V_{\mathrm{OC}}, J_{\mathrm{SC}}$ and $\eta$ values being observed when methyl groups are used. The EQE spectra for these dyes (Fig. 20b) are also consistent with this performance ordering, and we again (compare red curves in Fig. 17b and 20b) observe extension of the EQE spectrum of the dye containing 7 to lower energy compared to the remaining dyes.

One of the most dramatic differences between performances of DSCs assembled using acetone or $\mathrm{CH}_{2} \mathrm{Cl}_{2}$ copper(I) complex solutions is seen with the $6,6^{\prime}$-diphenyl substituted ancillary ligands. The performance of DSCs containing $[\mathrm{Cu}(\mathbf{1 3})(5)]^{+}$(firstgeneration, phenyl) improves when $\mathrm{CH}_{2} \mathrm{Cl}_{2}$ is used instead of acetone. For the $\mathrm{CH}_{2} \mathrm{Cl}_{2}$-derived dyes, a ripening effect is observed (Table S3 $\dagger$ ) with $\eta$ increasing from 1.85 to $2.06 \%$ (25.0 to $26.0 \%$ relative to N719), mainly due to an increase in $V_{\mathrm{OC}}$ from 492 to $537 \mathrm{mV}$ (Fig. S6 $\dagger$ ). For the $\mathrm{CH}_{2} \mathrm{Cl}_{2}$ dye solutions, $[\mathrm{Cu}(\mathbf{1 3})(5)]^{+}$is the optimum dye. However, extending the hole transporting domain appears to be unfavourable with losses in both $V_{\mathrm{OC}}$ and $J_{\text {SC }}$ (Fig. 18 versus 19, purple curves). The values of $\mathrm{EQE}_{\max }$ are $42.2 \%$ (at $\lambda_{\max }=470 \mathrm{~nm}$ ) for $[\mathrm{Cu}(13)(5)]^{+}$and $18.0 \%$ (at $\lambda_{\max }=480 \mathrm{~nm}$ ) for $[\mathrm{Cu}(\mathbf{1 3})(\mathbf{1 1})]^{+}$. DSCs with $[\mathrm{Cu}(\mathbf{1 3})(5)]^{+}$ (first-generation, phenyl) show similar values of $V_{\mathrm{OC}}, J_{\mathrm{SC}}$ and $\eta$ to those with $[\mathrm{Cu}(\mathbf{1 3})(\mathbf{1})]^{+}$(first-generation, methyl). We attribute the loss in performance on going from $[\mathrm{Cu}(\mathbf{1 3})(5)]^{+}$to $[\mathrm{Cu}(\mathbf{1 3})(\mathbf{1 1})]^{+}$to dye aggregation arising from intermolecular

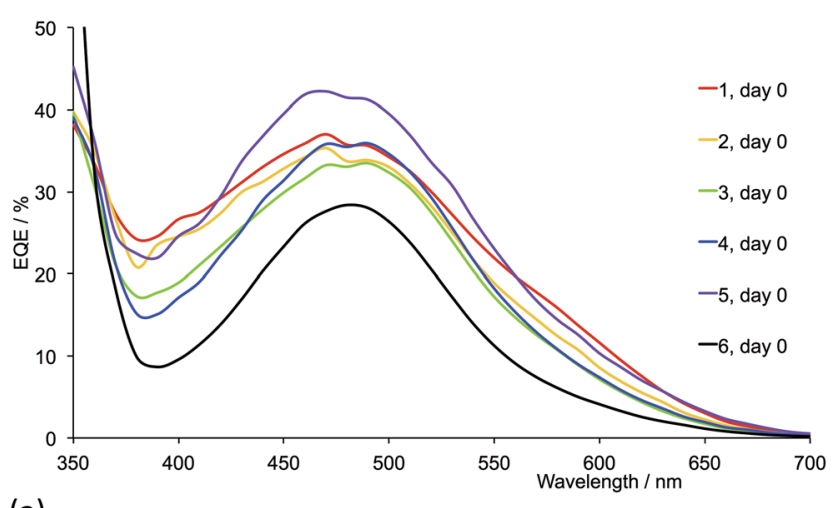

(a)

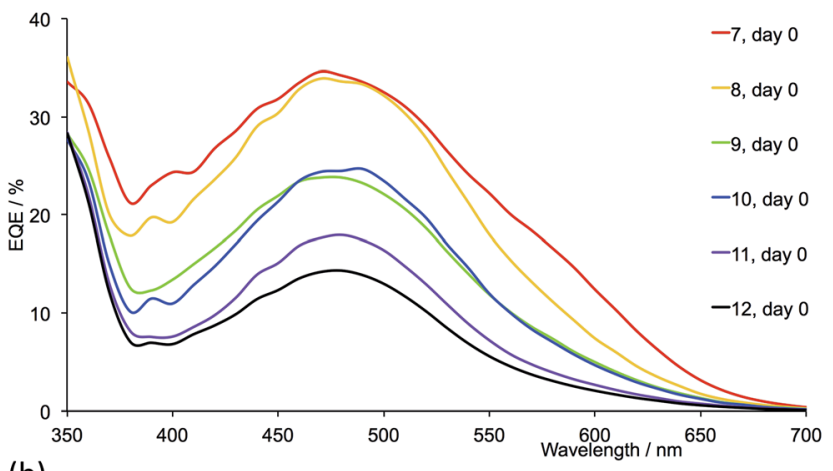

(b)

Fig. 20 EQE spectra (on the day of sealing) of DSCs with dyes containing ligands (a) 1-6 and (b) 7-12. $\mathrm{CH}_{2} \mathrm{Cl}_{2}$ was used in the $\left[\mathrm{CuL}_{2}\right]\left[\mathrm{PF}_{6}\right]$ dipping cycle. interactions involving the phenyl substituents and dendron in the second-generation 11.

\section{Conclusions}

We have prepared and characterized two series of homoleptic $\left[\mathrm{CuL}_{2}\right]^{+}$complexes containing bpy-derived ligands with different 6,6'-substituents, and either first-generation (ligands 1-6) or second-generation (ligands 7-12) hole transporting dendrons. $\mathrm{FTO} / \mathrm{TiO}_{2}$ electrodes were functionalized with the phosphonic acid 13. Ligand exchange using either $\mathrm{CH}_{2} \mathrm{Cl}_{2}$ or acetone solutions of $\left[\mathrm{CuL}_{2}\right]^{+}$gave two series of surface-bound heteroleptic dyes. Solid-state absorption spectra of dyecovered electrodes show that uptake of the dye is improved if acetone rather than $\mathrm{CH}_{2} \mathrm{Cl}_{2}$ is used in the dye-dipping cycle.

When acetone is used in the dye-soaking process, the best DSC efficiencies are obtained using the second generation dyes except for those with 2-naphthyl groups where low $J_{\mathrm{SC}}$ values contribute to a poor performance. The enhancement on going from first- to second-generation dendron is consistent with increased absorbance in the solid-state absorption spectra. The dyes that perform most efficiently, both for first and generationancillary ligands, are those with the methyl or $n$-butyl substituents. The EQE spectra of the DSCs containing the methylcontaining ancillary ligands show extension of the band to lower energy, consistent with electron injection over a wider range of wavelengths compared to the other dyes.

Overall, the DSC performances for cells made using acetone in the dye-dipping cycle are better than those made using $\mathrm{CH}_{2} \mathrm{Cl}_{2}$ solutions. For dyes assembled in $\mathrm{CH}_{2} \mathrm{Cl}_{2}$, those with ancillary ligands 1 and 7 (methyl groups) perform well, and exhibit the highest $V_{\mathrm{OC}}, J_{\mathrm{SC}}$ and $\eta$ values as well as EQE spectra that extend further towards higher wavelengths than the other dyes. The EQE spectra indicate that dyes containing the 2-naphthyl groups in $\mathrm{L}_{\text {ancillary }}$ show the poorest electron injection, consistent with low $J_{\mathrm{SC}}$ values. We conclude that incorporation of these large aromatic substituents is detrimental to DSC performance. In contrast, the first-generation 6,6'-diphenyl-substituted ancillary ligand, $\mathbf{5}$, gives a dye that performs as well as that containing $\mathbf{1}$ (methyl groups), and for $\mathrm{CH}_{2} \mathrm{Cl}_{2}$-derived dyes, $[\mathrm{Cu}(\mathbf{1 3})(5)]^{+}$is the optimum dye. However, the performance dramatically falls on going to the second-generation analogue $\mathbf{1 1}$.

Our overall conclusions are as follows. Among the $\left[\mathrm{Cu}(\mathbf{1 3})\left(\mathrm{L}_{\text {ancillary }}\right)\right]^{+}$dyes studied, the most promising $\mathrm{L}_{\text {ancillary }}$ ligands are $\mathbf{1}$ and $\mathbf{5}$ (methyl or phenyl substituents) if $\mathrm{CH}_{2} \mathrm{Cl}_{2}$ is used in the dye-dipping cycle. Generally, acetone is favoured over $\mathrm{CH}_{2} \mathrm{Cl}_{2}$ in the dye-assembly process, and gives adsorbed dyes that exhibit higher absorbances in the solid-state absorption spectra and enhanced conversion efficiencies. Use of the bulky 2-naphthyl substituents militates against good DSC performance. We will report on the effects of adding the coadsorbant cheno to these DSC systems in the near future.

\section{Acknowledgements}

The European Research Council (Advanced Grant 267816 LiLo), Swiss National Science Foundation and University of Basel are 
acknowledged for financial support. Cathrin Ertl, Roché Walliser and Dr Colin Martin are thanked for recording 500 NMR spectra, and Dr Collin Morris is acknowledged for recording the SEM image at the ZMB (University of Basel).

\section{Notes and references}

1 G. C. Vougioukalakis, A. I. Philippopoulos, T. Stergiopoulos and P. Falaras, Coord. Chem. Rev., 2011, 255, 2602, and references therein.

2 N. Armaroli, Chem. Soc. Rev., 2001, 30, 113.

3 N. Armaroli, Top. Curr. Chem., 2007, 280, 69.

4 N. Alonso-Vante, J-F. Nierengarten and J.-P. Sauvage, J. Chem. Soc. Dalton Trans., 1994, 1650.

5 N. Robertson, ChemSusChem, 2008, 1, 977.

6 B. Bozic-Weber, E. C. Constable and C. E. Housecroft, Chem. Soc. Rev., 2013, 257, 3089.

7 M. Sandroni, L. Favereau, A. Planchat, H. Akdas-Kilig, N. Szuwarski, Y. Pellegrin, E. Blart, H. Le Bozec, M. Boujtita and F. Odobel, J. Mater. Chem. A, 2014, 2, 9944.

8 B. Bozic-Weber, S. Brauchli, E. C. Constable, S. O. Fürer, C. E. Housecroft and I. A. Wright, Phys. Chem. Chem. Phys, 2013, 15, 4500 .

9 B. Bozic-Weber, E. C. Constable, C. E. Housecroft, P. Kopecky, M. Neuburger and J. A. Zampese, Dalton Trans., 2011, 40, 12584.

10 B. Bozic-Weber, S. Y. Brauchli, E. C. Constable, S. O. Fürer, C. E. Housecroft, F. J. Malzner, I. A. Wright and J. A. Zampese, Dalton Trans., 2013, 42, 12293.

11 G. J. Kubas, Inorg. Synth., 1990, 28, 68.

12 Bruker Analytical X-ray Systems, Inc., APEX2, version 2 User Manual, M86-E01078, Madison, WI, 2006.
13 G. M. Sheldrick, Acta Crystallogr., Sect. A: Cryst. Phys., Diffr., Theor. Gen. Crystallogr., 2008, 64, 112.

14 I. J. Bruno, J. C. Cole, P. R. Edgington, M. K. Kessler, C. F. Macrae, P. McCabe, J. Pearson and R. Taylor, Acta Crystallogr., Sect. B: Struct. Sci., 2002, 58, 389.

15 C. F. Macrae, I. J. Bruno, J. A. Chisholm, P. R. Edgington, P. McCabe, E. Pidcock, L. Rodriguez-Monge, R. Taylor, J. van de Streek and P. A. Wood, J. Appl. Crystallogr., 2008, 41, 466 .

16 S. Ito, P. Chen, P. Comte, M. K. Nazeeruddin, P. Liska, P. Péchy and M. Grätzel, Prog. Photovoltaics, 2007, 15, 603.

17 S. Ito, T. N. Murakami, P. Comte, P. Liska, C. Grätzel, M. K. Nazeeruddin and M. Grätzel, Thin Solid Films, 2008, 516, 4613.

18 F. Krönhke, Synthesis, 1976, 1.

19 See for example: P. V. James, K. Yoosaf, J. Kumar, K. G. Thomas, A. Listorti, G. Accorsi and N. Armaroli, Photochem. Photobiol. Sci., 2009, 8, 1432.

20 B. Bozic-Weber, V. Chaurin, E. C. Constable, C. E. Housecroft, M. Meuwly, M. Neuburger, J. A. Rudd, E. Schönhofer and L. Siegfried, Dalton Trans., 2012, 41, 14157.

21 M. K. Eggleston, D. R. McMillin, K. S. Koenig and A. J. Pallenberg, Inorg. Chem., 1997, 36, 172.

22 R. J. P. Williams, Eur. J. Biochem., 1995, 234, 363.

23 Y. Jahng, J. Hazelrigg, D. Kimball, E. Riesgo, R. Wu and R. P. Thummel, Inorg. Chem., 1997, 36, 5390.

24 D. V. Scaltrito, D. W. Thompson, J. A. O'Callaghan and G. J. Meyer, Coord. Chem. Rev., 2000, 208, 243.

25 T. E. Hewat, L. J. Yellowlees and N. Robertson, Dalton Trans., 2014, 43, 4127.

26 H. J. Snaith, Energy Environ. Sci., 2012, 5, 6513. 